\title{
SUPERPROCESSES AND THEIR LINEAR ADDITIVE FUNCTIONALS
}

\author{
E. B. DYNKIN
}

\begin{abstract}
Let $X=\left(X_{t}, P\right)$ be a measure-valued stochastic process. Linear functionals of $X$ are the elements of the minimal closed subspace $L$ of $L^{2}(P)$ which contains all $X_{t}(B)$ with $\int X_{t}(B)^{2} d P<\infty$. Various classes of $L$-valued additive functionals are investigated for measure-valued Markov processes introduced by Watanabe and Dawson. We represent such functionals in terms of stochastic integrals and we derive integral and differential equations for their Laplace transforms. For an important particular case-“weighted occupation times"-such equations have been established earlier by Iscoe.

We consider Markov processes with nonstationary transition functions to reveal better the principal role of the backward equations. This is especially helpful when we derive the formula for the Laplace transforms.
\end{abstract}

\section{INTRODUCTION}

1.1. With every measurable space $(E, \mathscr{B})$ another measurable space $(\mathscr{M}, \mathscr{B} \mathscr{M})$ is associated: $\mathscr{M}$ is the space of finite measures on $(E, \mathscr{B}), \mathscr{B} \mathscr{M}$ is the $\sigma$ algebra in $\mathscr{M}$ generated by the functions $F(\mu)=\mu(B)$ with $B \in \mathscr{B}$. Starting from a Markov transition function $p$ in $(E, \mathscr{B})$, we construct a Markov transition function $q$ in $(\mathscr{M}, \mathscr{B} \mathscr{\mathscr { N }})$ which we call the supertransition function over $p$. Let $\xi=\left(\xi_{t}, \Pi\right)$ be a Markov process with the transition function $p$ and $X=\left(X_{t}, P\right)$ be a Markov process with the transition function $q$. We say that $X$ is a superprocess over $\xi$ if $\Pi\left\{\xi_{t} \in B\right\}=P X_{t}(B)$ ( $P Y$ means $\left.\int Y d P\right)$. $X$ can be obtained by a passage to the limit from a system of many particles of small mass moving independently of each other according to the law of $\xi$ and dying or duplicating, with probability $\frac{1}{2}$, after each small fixed interval of time (see more precise description in Dynkin (1988)). We construct the superprocesses directly without any passage to the limit.

We fix a time interval $\Delta$ (open, closed or semiopen, finite or infinite) and we assume that $(E, \mathscr{B})$ is a standard Borel space and that

1.1.A. For every $B \in \mathscr{B}, p(r, x ; u, B)$ is $\mathscr{B}_{\Delta} \times \mathscr{B} \times \mathscr{B}_{\Delta}$-measurable in $s, x, u$ where $\mathscr{B}_{\Delta}$ is the Borel $\sigma$-algebra in $\Delta$.

Received by the editors November 18, 1987.

1980 Mathematics Subject Classification (1985 Revision). Primary 60G57, 60H05, 60J25, 60J55; Secondary $60 \mathrm{~J} 60,60 \mathrm{~J} 80$.

Key words and phrases. Measure-valued Markov processes, additive funtionals, stochastic integrals.

Partially supported by National Science Foundation Grant DMS 85-05020 and by the US Army Research Office through Mathematical Sciences Institute at Cornell University. 
The Markov semigroup associated with $p$ acts in the space $B^{+}$of bounded positive $\mathscr{B}$-measurable functions by the formula

$$
T_{u}^{r} f(x)=\int_{E} p(r, x ; u, d y) f(y) .
$$

We start with constructing another semigroup $V_{u}^{r}$ in $B^{+}$which is connected with $T_{u}^{r}$ by the equation

$$
V_{u}^{r} f=-\int T_{s}^{r}\left[\left(V_{u}^{s} f\right)^{2}\right] d s+T_{u}^{r} f, \quad r<u \in \Delta .
$$

Equation (1.2) determines the operators $V_{u}^{r}$ uniquely. Similarly to $T_{u}^{r}$, they are contractions (in the uniform norm); however, in contrast to $T_{u}^{r}$, they are nonlinear. The supertransition function $q$ is determined by the formula

$$
\int q(r, \mu ; u, d \nu) \exp \{-\langle f, \nu\rangle\}=\exp \left\{-\left\langle V_{u}^{r} f, \mu\right\rangle\right\}, \quad f \in B^{+}
$$

$\left(\langle f, \nu\rangle\right.$ means $\left.\int f d \nu\right)$.

After necessary tools are prepared in $\S 2$, we realize this program in $\S 3$ using arguments in Watanabe (1968) which we adjust to nonstationary transition functions in Borel spaces (Watanabe considered only stationary Feller transition functions). A certain simplication is possible because we deal only with a special type of "continuous state branching processes" studied by Watanabe.

1.2. If $p$ is the transition function of a diffusion process governed by the (backward) parabolic differential equation

$$
\partial F / \partial r=-L_{r} F
$$

( $L_{r}$ is an elliptic differential operator with coefficients depending on $r$ ) and if $f \in C^{2}$, then $v^{r}=V_{u}^{r} f$ is the solution of the differential equation

$$
\partial v / \partial r=-L_{r} v+v^{2}
$$

on the interval $\Delta_{\leq u}=\{r: r \leq u, r \in \Delta\}$ with the boundary condition $v^{u}=f$.

1.3. Put

$$
(\varphi * \psi)_{u}^{r}=\int_{r}^{u} T_{s}^{r}\left(\varphi_{u}^{s} \psi_{u}^{s}\right) d s
$$

In this notation, the equation (1.2) can be rewritten as follows

$$
v=-v * v+\varphi
$$

where

$$
\varphi_{u}^{r}=T_{u}^{r} f
$$

A formal solution of (1.7) is given by the series

$$
v=\sum_{n=1}^{\infty}(-1)^{n-1} \varphi^{n *}
$$


Here

$$
\varphi^{1 *}=\varphi ; \quad \varphi^{n *}=\sum_{k=1}^{n-1} \varphi^{k *} * \varphi^{(n-k) *} \text { for } n>1
$$

For every $\varphi$ (not necessarily positive), series (1.9) is dominated by the numerical series

$$
\Sigma C_{n}|\Delta|^{n-1}\|\varphi\|^{n}
$$

where $\Sigma C_{n} \theta^{n}=\frac{1}{2}-\frac{1}{2} \sqrt{1-4 \theta}$. Let $\|\varphi\|$ be the supremum of $\left|\varphi_{u}^{r}(x)\right|$ over all $x \in E, r<u \in \Delta$, and let $|\Delta|$ mean the length of $\Delta$. For every $0<\rho<$ $(4|\Delta|)^{-1}$, there exist constants $c_{\rho}>0$ and $0<\beta<1$ such that

$$
\left\|\varphi^{n *}\right\|<c_{\rho} \beta^{n}
$$

for all $\varphi$ such that $\|\varphi\| \leq \rho$. Thus series (1.9) converges absolutely and uniformly if $4\|\varphi\||\Delta|<1$. [A similar series has been used by Dawson (1977) for constructing stationary superprocesses.]

1.4. It follows from (1.3) that, for all $r<u \in \Delta, \mu \in \mathscr{M}, f \in B^{+}, \lambda \geq 0$,

$$
P_{r, \mu} \exp \left[-\lambda\left\langle f, X_{u}\right\rangle\right]=\exp \left[-\left\langle V_{u}^{r}(\lambda f), \mu\right\rangle\right]
$$

where $P_{r, \mu}$ are the transition probabilities corresponding to the transition function $q$ [i.e., $P_{r, \mu}\left\{X_{u} \in C\right\}=q(r, \mu ; u, C)$ ]. We conclude from (1.12) that

$$
\exp \left[-\left\langle V_{u}^{r}(\lambda f), \mu\right\rangle\right]=\sum_{n=0}^{\infty} \frac{(-\lambda)^{n}}{n !} a_{n}(f, \mu)
$$

with the power series convergent for $|\lambda|<(4\|f\||\Delta|)^{-1}$. If $f \geq 0$, then by (1.13)

$$
P_{r, \mu}\left[\left\langle f, X_{u}\right\rangle^{n}\right]=a_{n}(f, \mu) .
$$

One can extend this formula to all bounded measurable $f$. [An intermediate step is to apply (1.13a) to $f=\lambda_{1} f_{1}+\cdots+\lambda_{n} f_{n}$ and to compare the coefficients at $\lambda_{1} \cdots \lambda_{n}$ in both sides; cf. Dynkin (1988), Theorem 1.1.] Therefore (1.13) holds for $4|\lambda|\|f\||\Delta|<1$ even if $f$ is not positive.

An explicit expression for $a_{n}(f, \mu)$ is given in Dynkin (1988). Here we need only the formulae for $n=1$ and 2 . It follows from (1.9) that $v_{u}^{r}(\lambda)=V_{u}^{r}(\lambda f)$ has the form

$$
v(\lambda)=\lambda \varphi-\lambda^{2} \varphi * \varphi+O\left(\lambda^{3}\right) \text { as } \lambda \downarrow 0
$$

(with $\varphi$ defined by (1.8)). By (1.13) and (1.14),

$$
P_{r, \mu}\left\langle f, X_{u}\right\rangle=\left\langle T_{u}^{r} f, \mu\right\rangle
$$

and

$$
\begin{aligned}
P_{r, \mu}\left[\left\langle f, X_{u}\right\rangle^{2}\right] & =\left[P_{r, \mu}\left\langle f, X_{u}\right\rangle\right]^{2}+2\left\langle(\varphi * \varphi)_{u}^{r}, \mu\right\rangle \\
& =\left\langle T_{u}^{r} f, \mu\right\rangle^{2}+2 \int_{r}^{u}\left\langle T_{s}^{r}\left(\varphi_{u}^{s}\right)^{2}, \mu\right\rangle d s .
\end{aligned}
$$


Let $X=\left(X_{t}, \mathscr{F}_{t}, P\right)$ be a Markov process with transition function $q$. It follows from (1.15), (1.16) that

$$
P\left[\left(\left\langle f, X_{u}\right\rangle-P\left\{\left\langle f, X_{u}\right\rangle \mid F_{r}\right\}\right)^{2}\right]=2 \int_{r}^{u}\left\langle\left(\varphi_{u}^{s}\right)^{2}, \mu_{s}\right\rangle d s=2 \int \varphi_{u}^{s}(x)^{2} \mu_{u}^{r}(d s, d x)
$$

where

$$
\mu_{s}(B)=P X_{s}(B)
$$

and $\mu_{u}^{r}$ is the restriction to $[r, u] \times E$ of the measure

$$
\mu(d s, d x)=d s \mu_{s}(d x) \text {. }
$$

Note that

$$
\mu_{t}=\mu_{r} T_{t}^{r} \text { for } r<t \in \Delta \text {, }
$$

i.e., that $\left\{\mu_{s}\right\}$ is an entrance law for $p(s, x ; t, d y)$. Therefore there exists a Markov process $\xi=\left(\xi_{t}, \Pi\right)$ with the transition function $p$ such that $\Pi\left\{\xi_{t} \in B\right\}=\mu_{t}(B)$ for all $t \in \Delta, B \in \mathscr{B}$, or, in other words, such that $X$ is the superprocess over $\xi$. If $\xi_{s}$ is a measurable process, then formula (1.17) can be rewritten in the form

$$
P\left[\left(\left\langle f, X_{u}\right\rangle-P\left\{\left\langle f, X_{u}\right\rangle \mid \mathscr{F}_{r}\right\}\right)^{2}\right]=2 \Pi \int_{r}^{u} \varphi_{u}^{s}\left(\xi_{s}\right)^{2} d s .
$$

1.5. If $X$ is a superprocess, then $\left\langle f, X_{t}\right\rangle$ belongs to $L^{2}(P)$ for all $f \in B^{+}$. We denote by $L_{u}^{r}(P)$ the minimal closed subspace of $L^{2}(P)$ which contains $\left\langle f, X_{t}\right\rangle$ for all $f \in B^{+}, t \in[r, u]$. A linear additive functional of $X$ is a family of random variables $A_{u}^{r}, r<u \in \Delta$, with the following properties:

1.5.A. $A_{u}^{r}$ belongs to $L_{u}^{r}(P)$.

1.5.B. For every $r<s<u \in \Delta, A_{s}^{r}+A_{u}^{s}=A_{u}^{r} P$-a.s.

(We consider only square-integrable functionals. Of course, the definition of $L_{u}^{r}$ can be modified with convergence in probability substituted for $L^{2}$ convergence.)

Two functionals $A$ and $B$ are called equivalent if, for every $r<u \in \Delta$, $A_{u}^{r}=B_{u}^{r} \quad P$-a.s. Classes of equivalent linear additive functionals form a linear space $\mathscr{L}$ with a family of Hilbert seminorms

$$
\|A\|_{u}^{r}=\left\{P\left[\left(A_{u}^{r}\right)^{2}\right]\right\}^{1 / 2} .
$$

We say that an additive functional $A$ is fair and we write $A \in \mathscr{L}_{f}$ if

$$
P\left\{A_{u}^{r} \mid \mathscr{F}_{r}\right\}=0 \text { for every } r<u \in \Delta \text {. }
$$

A functional

$$
A_{u}^{r}=\left\langle h^{u}, X_{u}\right\rangle-\left\langle h^{r}, X_{r}\right\rangle
$$

is fair if

$$
T_{u}^{r} h^{u}=h^{r} \quad \text { for all } r<u \in \Delta \text {, }
$$


i.e., if $h$ is an exit law. [We drop the condition $h \geq 0$ which usually is included into the definition of an exit law.]

If $A$ is fair, then

$$
P\left\{A_{u}^{r} \mid \mathscr{F}_{t}\right\}=A_{t}^{r} P \text {-a.s. for all } r<t<u \in \Delta .
$$

Hence $\left(A_{t}^{r}, \mathscr{F}_{t}, P\right)$ is a martingale on $\Delta_{\geq r}=\{t: t \geq r, t \in \Delta\}$. If $A, B \in \mathscr{L}_{f}$, then

$$
P A_{t}^{r} B_{u}^{s}= \begin{cases}0 & \text { if }[r, t] \cap[s, u]=\varnothing, \\ P A_{\beta}^{\alpha} B_{\beta}^{\alpha} & \text { if }[r, t] \cap[s, u]=[\alpha, \beta] .\end{cases}
$$

1.6. We denote by $\mathscr{H}=\mathscr{H}(P)$ the space of $\mathscr{B}{ }_{\Delta} \times \mathscr{B}$-measurable functions $h$ such that, for all $r<u \in \Delta$,

$$
\|h\|_{u}^{r}=\left(\int h^{s}(x)^{2} \mu_{u}^{r}(d s, d x)\right)^{1 / 2}<\infty .
$$

$\mathscr{H}$ is a linear topological space with the topology determined by the family of seminorms (1.28). (Note that, if $X_{t}(\omega)$ is measurable, then

$$
\left.\|h\|_{u}^{r}=\left(P \int_{r}^{u}\left\langle\left(h^{s}\right)^{2}, X_{s}\right\rangle d s\right)^{1 / 2} .\right)
$$

Let $\mathscr{R}$ stand for the set of all bounded $\mathscr{B}_{\Delta} \times \mathscr{B}$-measurable functions $h$ such that $h^{s}\left(\xi_{s}\right)$ is right continuous in $s$ on $\Delta$ for every $\omega$, and let $\mathscr{C}$ be the $\sigma$-algebra in $\Delta \times E$ generated by $\mathscr{R}$. [Note that, if $h$ is $\mathscr{C}$-measurable, then $h^{s}\left(\xi_{s}\right)$ is optional relative to the filtration generated by $\xi$.] Put $h \in \mathscr{K}$ if $h$ is $\mathscr{C}$-measurable and it belongs to $\mathscr{H}$.

We say that a function $h: \Delta \times E \rightarrow \mathbf{R}$ is a broken exit law if, for some $t_{0}<t_{1}<\cdots<t_{n} \in \Delta, h$ is an exit law on each of the intervals $\Delta_{\leq t_{0}}$, $\left(t_{0}, t_{1}\right], \ldots,\left(t_{n-1}, t_{n}\right], \Delta_{>t_{n}}$. In $\S 4$ we prove that the set $\mathscr{E}$ of all bounded broken exit laws is everywhere dense in $\mathscr{K}$ and we construct a linear mapping $I$ from $\mathscr{K}$ onto $\mathscr{L}_{f}$ such that:

1.6.A. If $h$ is an exit law on $(r, u]$, then

$$
I_{u}^{r}(h)=\left\langle h^{u}, X_{u}\right\rangle-\left\langle h^{r}, X_{r}\right\rangle .
$$

1.6.B. For every $r<u \in \Delta$ and for all $h, \tilde{h} \in \mathscr{K}$,

$$
\begin{aligned}
P I_{u}^{r}(h) I_{u}^{r}(\tilde{h}) & =\int h^{s}(x) \tilde{h}^{s}(x) \mu_{u}^{r}(d s, d x) \\
& \left(=P \int_{r}^{u}\left\langle h^{s} \tilde{h}^{s}, X_{s}\right\rangle d s \text { if } X_{s} \text { is measurable }\right) .
\end{aligned}
$$

Conditions 1.6.A-B determine the mapping $I$ uniquely. We use the following notation

$$
I_{u}^{r}(h)=\int_{r}^{u} h^{s} d Z_{s}
$$


1.7. For every $\widetilde{\Delta} \subset \Delta$, we denote by $\mathscr{F}(\widetilde{\Delta})$ the $\sigma$-algebra in $\Omega$ generated by $X_{t}, t \in \tilde{\Delta}$. We put $\mathscr{F}[s, u]=\mathscr{F}([s, u])$ and $\mathscr{F}_{\geq s}=\mathscr{F}\left(\Delta_{\geq s}\right)$. Let $\mathscr{P}_{s}$ be the class of all probability measures on $\mathscr{F}_{\geq s}$ such that $\left(X_{t}, \mathscr{F}[s, t], P\right)_{t \in \Delta_{>s}}$ is a Markov process with the transition function $q$. Let $h$ be a $\mathscr{B} \Delta \times \mathscr{B}$ measurable function. Put $P \in \mathscr{P}_{s}(h)$ if $P \in \mathscr{P}_{s}$ and if condition (1.28) holds for all $r<u \in \Delta_{\geq s}$. If $h$ is bounded, then $\mathscr{P}_{s}(h)=\mathscr{P}_{s}$ for all $s$.

For every $\mathscr{C}$-measurable function $h$, we define a canonical version of $I(h)$ such that, for all $s \in \Delta, P \in \mathscr{P}_{s}(h)$, the restriction of $I_{u}^{r}(h)$ ot $\Delta_{\geq s}$ is a fair linear additive functional of $\left(X_{t}, \mathscr{F}[s, t], P\right)$. We denote by $\mathscr{Z}$ the family of these Markov processes and we say that $I(h)$ is a fair additive functional of $\mathscr{X}$.

Suppose that:

1.7.A. For every $r<u \in \Delta, f \in B^{+}, P \in \mathscr{P}_{r}$, the function $P_{s, X_{s}}\left\langle f, X_{u}\right\rangle$ is right continuous in $s$ on $[r, u) \quad P$-a.s.

Then the canonical version of $I_{u}^{s}(h)$ is continuous in $u$ on $\Delta_{\geq_{s}} P$-a.s. for every $s \in \Delta$ and every $P \in \mathscr{P}_{s}$. This result is proved in $\S 7$. A preliminary weaker result with continuity replaced by right continuity is proved in $\S 4$. [For instance, 1.7.A holds if $X$ is a right process. See the definition in $\S 8.5$. Recently the author proved that, if $\xi$ is a right process, then $X$ can be chosen to be right too.]

Assume that $\xi_{s}$ has the following property:

1.7.B. $\sigma$-algebra $\mathscr{B}_{\Delta} \times \mathscr{B}$ is generated by $\mathscr{R}$.

[This is true, for instance, for every right continuous process in a topological state space $E$.] In this situation $\mathscr{C}=\mathscr{B}_{\Delta} \times \mathscr{B}, \mathscr{K}=\mathscr{H}$ and (1.31) can be interpreted as a stochastic integral with respect to a martingale measure. Indeed $Z_{t}(B)=I\left(1_{\Delta_{\leq 1}} 1_{B}\right)$ is a stochastic measure for fixed $t$ (i.e., $Z_{t}\left(\cup B_{n}\right)=\Sigma Z_{t}\left(B_{n}\right)$ in $L^{2}(P)$ for disjoint $\left.B_{1}, \ldots, B_{n}, \ldots\right)$ and it is a martingale (relative to $\mathscr{F}_{t}, P$ ) for a fixed $B$. [Stochastic integrals with respect to more general martingale measures have been introduced in Walsh (1986).]

1.8. The processes $\xi$ and $X$ can be chosen to satisfy 1.7.A, B if $p(r, x ; u, B)=$ $p_{u-r}(x, B)$ is a stationary transition function in a separable locally compact metric space and if the corresponding semigroup $T_{t}$ preserves the space $C_{0}$ of continuous functions tending to 0 at infinity and is strongly continuous in $C_{0}$. This follows from more general criterion for nonstationary transition functions $p$ proved in $\S 8$.

1.9. We denote by $\mathscr{N}$ the set of all linear additive functional $A$ of $\mathscr{X}$ such that:

1.9.A. $A_{u}^{r} \geq 0$ for all $\omega$.

1.9.B. For every $\omega$ and every $r, A_{u}^{r}$ is right continuous in $u$ on $\Delta_{>r}$.

1.9.C. For each $\omega, A_{u}^{r} \rightarrow 0$ as $u \downarrow r$.

We call elements of $\mathscr{N}$ positive linear additive functionals (though conditions 1.9.B,C have nothing to do with positivity). It follows from 1.9.A-C that, for 
every $r \in \Delta$, there exists a random measure $M_{r}$ on $\Delta_{\geq r}$ such that

$$
M_{r}(r, u]=A_{u}^{r} \quad \text { for every } u \in \Delta_{\geq r} \text { and all } \omega \text {. }
$$

For every $u_{1}<u_{2} \in \Delta_{\geq r}$ and $P \in \mathscr{P}_{r}$,

$$
M_{r}\left(u_{1}, u_{2}\right]=A_{u_{2}}^{u_{1}} \quad P \text {-a.s. }
$$

and therefore, for arbitrary $r_{1}<r_{2} \in \Delta$ and $P \in \mathscr{P}_{r_{1}}$, the restriction of $M_{r_{1}}$ to $\Delta_{\geq r_{2}}$ coincides with $M_{r_{2}} P$-a.s.

We define the characteristic of $A$ by the formula

$$
h^{s}(x)=P_{s, \delta_{x}} M_{s}\left(\Delta_{\geq s}\right)
$$

where $\delta_{x}$ is the unit measure concentrated at $x$. Obviously, the characteristics of equivalent functionals coincide. We consider only functionals with finite characteristics.

In $\S 5$ we show that:

1.9.A. The characteristic determines $A$ up to equivalence.

1.9.B. It is an exit rule which means:

$$
h \geq 0 ; \quad T_{u}^{r} h^{u} \leq h^{r} \quad \text { for all } r<u \in \Delta ; \quad T_{u}^{r} h^{u} \uparrow h^{r} \text { as } u \downarrow r .
$$

1.9.C. It is a pure exit rule, i.e., if $g$ is an exit law and if $0 \leq g \leq h$, then $g=0$.

We also prove that, if 1.7.A,B are satisfied, then every bounded exit rule $h$ is the characteristic of a positive linear additive functional which can be represented by the formula

$$
A_{u}^{r}=\int_{r}^{u} h^{s} d Z_{s}+\left\langle h^{r}, X_{r}\right\rangle-\left\langle h^{u}, X_{u}\right\rangle .
$$

It has been proved in Dynkin (1972) that the formula

$$
h^{s}(x)=\int_{\Gamma} K(s, x ; \eta) \gamma(d \eta)
$$

establishes a 1-1 correspondence between all exit rules $h$ subject to the condition

$$
\sup _{s \in \Delta}\left\langle h^{s}, \mu_{s}\right\rangle<\infty
$$

and all finite measures $\gamma$ on a certain measurable space $\left(\Gamma, \mathscr{B}_{\Gamma}\right)$ (the exit space for $\xi$ ). Obviously, $h$ belongs to $\mathscr{H}$ if and only if, for all $r<u \in \Delta$,

$$
\int_{\Gamma \times \Gamma} N_{u}^{r}\left(\eta_{1}, \eta_{2}\right) \gamma\left(d \eta_{1}\right) \gamma\left(d \eta_{2}\right)<\infty
$$

where

$$
N_{u}^{r}\left(\eta_{1}, \eta_{2}\right)=\int_{\Delta \times E} K\left(s, x ; \eta_{1}\right) K\left(s, x ; \eta_{2}\right) \mu_{u}^{r}(d s, d x) .
$$

If $p(r, x ; u, d y)=p(r, x ; u, y) \lambda(d y)$ for some measure $\lambda$, then the density function $p(r, x ; u, y)$ can be chosen to satisfy the equation

$$
\int p(r, x ; t, y) \lambda(d y) p(t, y ; u, z)=p(s, x ; u, z)
$$

$$
\text { for all } r<t<u \in \Delta, x, z \in E
$$


[see Dynkin and Kuznetsov (1974)]. We put $p(r, x ; u, y)=0$ for $r \geq u$ and we note that

$$
h^{r}(x)=\int_{\Delta \times E} p(r, x ; t, y) \sigma(d t, d y)
$$

is an exit rule for every measure $\sigma$. A condition similar to (1.37) can be written in terms of $\sigma$ for $h$ to belong to $\mathscr{H}$.

The "weighted occupation time"

$$
A_{u}^{r}=\int_{r}^{u}\left\langle\rho^{s}, X_{s}\right\rangle d s
$$

corresponds to the exit rule

$$
h^{s}(x)=\int_{\Delta_{>s}} T_{u}^{s} \rho^{u} d u .
$$

The so-called "local occupation time" at a point $z$ investigated in Iscoe (1986a) [cf Dynkin (1988)] corresponds to the exit rule

$$
h^{s}(x)=\int_{\Delta_{>s}} p(s, x ; u, z) d u .
$$

1.10. One of central results of the present paper-an expression for the Laplace transform of $\int_{r}^{u} h^{s} d Z_{s}$-is proved in $\S 6$. We show that

$$
P_{r, \mu} \exp \int_{r}^{u} h^{s} d Z_{s}=\exp \left\langle w_{u}^{r}, \mu\right\rangle
$$

for all $r<u \in \Delta$ such that $u-r<(4\|h\|)^{-1}$. Here

$$
w=\sum_{n=2}^{\infty} \varphi^{n *} \text { with } \varphi_{u}^{r}=h^{r} 1_{r \leq u} .
$$

For every $u, v^{r}=h^{r}+w_{u}^{r}$ satisfies the equation

$$
v^{r}-\int_{r}^{u} T_{s}^{r}\left[\left(v^{s}\right)^{2}\right] d s=h^{r}, \quad r \in \Delta_{<u} .
$$

Moreover (1.39) holds on the interval $\left(r_{0}, u\right)$ where

$$
r_{0}=\inf \left\{t: \sup _{r \in[t, u]} \sup _{x} \log P_{r, \delta_{x}} \exp \int_{r}^{u} h^{s} d Z_{s}<\infty\right\} .
$$

We also prove that the uniform norm $\left\|v^{r}\right\|$ is bounded on every interval $[t, u) \subset$ $\left(r_{0}, u\right)$ but it is unbounded on $\left(r_{0}, u\right)$.

For the positive linear functional $A$ given by (1.36), it can be proved, in a similar way, that

$$
P_{r, \mu} \exp \left\{-A_{u}^{r}\right\}=\exp \left\{-\left\langle v^{r}, \mu\right\rangle\right\} \quad \text { for all } r<u \in \Delta
$$

where $v$ is the unique solution of the equation

$$
v^{r}+\int_{r}^{u} T_{s}^{r}\left[\left(v^{s}\right)^{2}\right] d s=h^{r}-T_{u}^{r} h^{u}, \quad r \in \Delta_{<u} .
$$


If $p$ is the fundamental solution of the diffusion equation (1.4) and if $A$ has the form (1.38) with a bounded continuous $\rho$, then (1.41a) is equivalent to the differential equation

$$
\partial v / \partial r=-L_{r} v+v^{2}-\rho^{r}, \quad r \in \Delta_{\leq u}
$$

with the boundary condition $v^{u}=0$. This equation (in a slightly different form) has been established, first, in Iscoe (1986).

1.11. In $\S 7$, by using formulae (1.39) and (1.41), we establish that, if $h$ is a bounded $\mathscr{B}_{\Delta \times E}$-measurable function, then for all sufficiently small $\alpha$,

$$
P_{r, \mu} \exp \left[\alpha A_{u}^{r}-\alpha^{2} B_{u}^{r} / 2\right]=1
$$

where

$$
A_{u}^{r}=\int_{r}^{u} h^{s} d Z_{s}, \quad B_{u}^{r}=2 \int_{r}^{u}\left\langle\left(h^{s}\right)^{2}, X_{s}\right\rangle d s .
$$

It follows from (1.44)-(1.45) that

$$
P\left\{\left(A_{u}^{r}\right)^{2} \mid \mathscr{F}_{r}\right\}=P\left\{B_{u}^{r} \mid \mathscr{F}_{r}\right\}
$$

and therefore $B_{t}^{r}$ is the quadratic variation for the martingale $\left(A_{t}^{r}, \mathscr{F}_{t}, P\right)$, $t \in \Delta_{>r}$. Formulae (1.44)-(1.45) are also used to prove the existence of a continuous version of $\int_{r}^{u} h^{s} d Z_{s}$.

Martingale properties of superprocesses have been studied by Roelly-Coppoletta (1986). In particular, she established (1.44) and (1.46) for a special class of martingales.

1.12. Suppose that $h_{n}$ is a sequence of real-valued functions. We say that $h_{n}$ converges to $h$ boundedly if $h_{n}$ are uniformly bounded and converge to $h$ pointwise. We use many times the following

Multiplicative Systems Theorem (Meyer (1966), Chapter 1, Theorem 20). Suppose that a linear space $H$ of bounded functions contains 1 and is closed under bounded convergence. If $H$ contains a family $Q$ which is closed under multiplication, then $H$ contains all bounded functions which are measurable relative to the $\sigma$-algebra generated by $Q$.

We also use the following version of this theorem:

Modified Multiplicative Systems Theorem. Let $Q$ be an algebra of bounded realvalued functions such that $1 \in Q$. Let $H$ be closed under bounded convergence. If $H \supset Q$, then $H$ contains all bounded functions which are measurable relative to the $\sigma$-algebra generated by $Q$.

To prove this statement, it is sufficient to apply the Multiplicative Systems Theorem to the maximal element $M$ of the class of all linear spaces $L$ such that $Q \subset L \subset H$. (The existence of such a maximal element follows from Zorn's lemma.) 
1.13. Acknowledgments. The author is deeply indebted to Patrick Fitzsimmons and Ronald Getoor who have read carefully the manuscript and suggested a number of corrections and improvements to it. In particular, they suggested a substantial simplification of the proof of Theorem 3.1. A number of corrections have been made also by Yuan-chung Sheu.

\section{Space of finite Measures}

2.1. Let $(\mathscr{K}, \mathscr{B} \mathscr{\mu})$ be the space of all finite measures on $(E, \mathscr{B})$. We say that $\mu$ is a subprobability measure and we put $\mu \in \mathscr{M}_{1}$ if $\mu(E) \leq 1$. We denote by $\mathfrak{M}_{1}$ the set of all subprobability measures on $\left(\mathscr{M}, \mathscr{B}_{\mathscr{M}}\right)$. To every $f \in B^{+}$ there corresponds a positive bounded $\mathscr{M}_{\mathscr{B}}$-measurable function

$$
F_{f}(\mu)=e^{-\langle f, \mu\rangle}, \quad \mu \in \mathscr{M} .
$$

Lemma 2.1. In every standard Borel space $(E, \mathscr{B})$ there exists a countable set $W \subset B^{+}$with the following properties:

2.1.A. If a set $H \supset W$ is closed under bounded convergence, then $H \supset B^{+}$.

2.1.B. If a linear space $\mathscr{H}$ of functions on $\mathscr{M}$ contains 1 and the functions (2.1) for all $f \in W$, then it contains all positive bounded $\mathscr{M}_{\mathscr{B}}$-measurable functions.

2.1.C. If $P_{n} \in \mathfrak{M}_{1}$ and if

$$
\int_{\mathscr{M}} F_{f}(\nu) P_{n}(d \nu) \rightarrow l(f) \quad \text { for all } f \in W,
$$

then there exists a $P \in \mathfrak{M}_{1}$ such that

$$
l(f)=\int_{\mathscr{M}} F_{f}(\nu) P(d \nu) .
$$

Proof. A metric $d(x, y)$ can be introduced into $E$ such that $E$ becomes a separable compact space and $\mathscr{B}$ coincides with the Borel $\sigma$-algebra. Let $C^{++}$ be the set of all strictly positive continuous functions on $E$ and let $W$ be a countable everywhere dense subset of $C^{++}$(with respect to uniform convergence).

To prove 2.1.A we put $h \in \widetilde{H}$ if $\max \{h, 0\}$ and $\max \{-h, 0\}$ belong to $H$. If $H \supset W$ is closed under bounded convergence, then $\widetilde{H}$ contains all continuous functions. It follows from the Modified Multiplicative Systems Theorem that $\widetilde{H}$ contains all bounded $\mathscr{B}$-measurable functions, and therefore $H \supset B^{+}$.

The family $F_{f}, f \in W$, is closed under multiplication and it generates $\mathscr{M}_{\mathscr{B}}$. Therefore 2.1.B follows from the Multiplicative Systems Theorem.

To get 2.1.C we consider a point $\partial$ not in $\mathscr{M}$ and put $\widetilde{\mathscr{M}}=\mathscr{M} \cup \partial$ with the topology defined by the following convention:

$$
\begin{array}{cc}
\mu_{n} \rightarrow \mu \in \mathscr{M} & \text { if }\left\langle f, \mu_{n}\right\rangle \rightarrow\langle f, \mu\rangle \text { for all } f \in W, \\
\mu_{n} \rightarrow \partial & \text { if }\left\langle f, \mu_{n}\right\rangle \rightarrow \infty \text { for all } f \in W .
\end{array}
$$


Note that $\mu_{n} \rightarrow \partial$ if and only if $\left\langle\mu_{n}, 1\right\rangle \rightarrow \infty$, and therefore $\widetilde{\mathscr{M}}$ is compact. Measures $P_{n} \in \mathfrak{M}_{1}$ can be continued to probability measures $\widetilde{P}_{n}$ on $\widetilde{\mathscr{M}}$. A subsequence $\widetilde{P}_{n_{k}}$ of the sequence $\widetilde{P}_{n}$ converges weakly to a probability measure $\widetilde{P}$. For every $f \in W$, the function

$$
\begin{gathered}
\widetilde{F}_{f}(\mu)=F_{f}(\mu) \text { for } \mu \in \mathscr{M}, \\
\widetilde{F}_{f}(\partial)=0
\end{gathered}
$$

is continuous on $\widetilde{\mathscr{M}}$ and therefore

$$
\int_{\mathscr{M}} F_{f}(\nu) P_{n}(d \nu)=\int_{\tilde{\mathscr{M}}} \tilde{F}_{f}(\nu) \tilde{P}_{n}(d \nu) \rightarrow \int_{\tilde{\mathscr{M}}} \tilde{F}_{f}(\nu) \widetilde{P}(d \nu)
$$

which proves that 2.1.C holds with $P$ equal to the restriction of $\widetilde{P}$ to $\mathscr{M}$.

2.2. We call

$$
L_{P}(f)=\int_{\mathscr{M}} e^{-\langle f, \nu\rangle} P(d \nu), \quad f \in B^{+},
$$

the Laplace functional for $P \in \mathfrak{M}_{1}$. By the Multiplicative Systems Theorem, $P$ is uniquely determined by $L_{P}$.

Let $W$ be the family described in Lemma 2.1. To every $P \in \mathfrak{M}_{1}$ there corresponds a function

$$
g_{P}(f)=-\log L_{P}(f), \quad f \in W,
$$

from $W$ to $[0,+\infty]$ that is an element of a compact topological space $\mathscr{D}=$ $[0,+\infty]^{W}$. Put $g \in \mathscr{G}$ if $g \in \mathscr{D}$ and if, for every $\alpha>0$, there exists a $P_{\alpha} \in \mathfrak{M}_{1}$ such that $g_{P_{a}}(f)=\alpha g(f)$ for all $f \in W$. Obviously $\mathscr{G}$ is closed under multiplication with positive numbers. If $P$ is the convolution of $P^{\prime}$ and $P^{\prime \prime}$, then $g_{P}(f)=g_{P^{\prime}}(f)+g_{P^{\prime \prime}}(f)$. Therefore $\mathscr{G}$ is closed under addition. If $g_{n} \in \mathscr{G}$ and if $g_{n} \rightarrow g$ in $\mathscr{D}$, then $g \in \mathscr{G}$. Indeed, $\exp \left[-\alpha g_{n}(f)\right]=$ $L_{P_{a, n}}(f)$ for some $P_{\alpha, n} \in \mathfrak{M}_{1}$, and, by 2.1.C, there exists $P_{\alpha} \in \mathfrak{M}_{1}$ such that $\exp [-\alpha g(f)]=L_{P_{n}}(f)$.

The set of finite measures $\sigma$ on $\mathscr{D}$ such that $\int g(f) \sigma(d g) \in \mathscr{G}$ is closed in the weak topology. It contains all finite measures whose support is a finite subset of $\mathscr{G}$. Hence it contains all finite measures concentrated on $\mathscr{G}$ (see, e.g., Watanabe (1968), p. 144). In other words, for every finite measure $\sigma$ on $\mathscr{G}$,

$$
\int_{\mathscr{G}} g \sigma(d g) \in \mathscr{G} \text {. }
$$

2.3. Let $V$ be a mapping from $B^{+}$to $B^{+}$. We write $V \in \mathscr{A}$ if there exists a Markov kernel $q(\mu, d \nu)$ such that

$$
\int_{\mathscr{M}} q(\mu, d \nu) e^{-\langle f, \nu\rangle}=e^{-\langle V f, \mu\rangle} \quad \text { for all } f \in B^{+}, \mu \in \mathscr{M} .
$$


Note that a mapping $V: B^{+} \rightarrow B^{+}$belongs to $\mathscr{A}$ if and only if the following three conditions are satisfied:

2.3.A. $V(0)=0$.

2.3.B. $V$ preserves bounded convergence.

2.3.C. For every $\mu \in \mathscr{M}$,

$$
g(f)=\langle V f, \mu\rangle
$$

belongs to $\mathscr{G}$.

Obviously conditions 2 .3.A and $C$ are necessary. If $V \in \mathscr{A}$ and if $f_{n} \rightarrow f$ boundedly, then $\left\langle V f_{n}, \mu\right\rangle \rightarrow\langle V f, \mu\rangle$ for every $\mu \in \mathscr{M}$ and therefore $V f_{n} \rightarrow$ $V f$ boundedly. Hence 2.3.B is also necessary. On the other hand, if 2.3.C holds, then for every $\mu \in \mathscr{M}$, there exists a subprobability measure $q(\mu, \cdot)$ such that (2.5) holds for all $f \in W$. By 2.3.B and 2.1.A, (2.5) is true for all $f \in B^{+}$, and by 2.3.A $q(\mu, \mathscr{M})=1$.

2.4. We say that $V$ is the $U$-limit of $V_{n}$ if, for every $c, \varepsilon>0$, there exists an $N$ such that $\left\|V_{n} f-V f\right\|<\varepsilon$ for all $n \geq N$ and all $f \in B^{+}$such that $\|f\| \leq c$. We need the following properties of $\mathscr{A}$ :

2.4.A. If $s \rightarrow V_{s}$ is a map from a measurable space $\left(S, \mathscr{B}_{S}\right)$ to $\mathscr{A}$ such that $V_{s} f(x)$ is $\mathscr{B} \times \mathscr{B}_{S}$-measurable and bounded for every $f \in B^{+}$and if $\gamma$ is a finite measure on $\mathscr{B}_{S}$, then

$$
V=\int_{S} V_{s} \gamma(d s) \in \mathscr{A} .
$$

2.4.B. $\mathscr{A}$ is closed under addition and multiplication by positive numbers.

2.4.C. $\mathscr{A}$ is closed under multiplication: if

$$
\begin{aligned}
& \int q_{1}(\mu, d \nu) e^{-\langle f, \nu\rangle}=e^{-\langle U f, \mu\rangle}, \\
& \int q_{2}(\nu, d \rho) e^{-\langle h, \rho\rangle}=e^{-\langle V h, \rho\rangle},
\end{aligned}
$$

then

$$
\int q_{1}(\mu, d \nu) q_{2}(\nu, d \rho) e^{-\langle h, \rho\rangle}=e^{-\langle U V h, \mu\rangle} .
$$

2.4.D. If $V$ is the $U$-limit of $V_{n} \in \mathscr{A}$, then $V \in \mathscr{A}$.

To prove 2.4.A, we consider the image $\sigma$ of $\gamma$ under the mapping (2.6) and we note that, by (2.4), for every $\mu \in \mathscr{M}$,

$$
\langle V f, \mu\rangle=\int_{S}\left\langle V_{s} f, \mu\right\rangle \gamma(d s)=\int_{\mathscr{G}} g(f) \sigma(d g) .
$$

2.4.B is a particular case of 2.4.A. To get 2.4.D, we use the fact that $\mathscr{G}$ is closed under pointwise convergence.

2.5. If $\Pi_{\mu}$ is the Poisson random measure with the intensity $\mu$, then

$$
E \exp \left\{-\left\langle f, \Pi_{\mu}\right\rangle\right\}=\exp \{-\langle V f, \mu\rangle\}
$$


with

$$
V f(x)=1-e^{-f(x)}
$$

Thus, for every $k>0$,

$$
E \exp \left\{-\left\langle f, k^{-1} \Pi_{2 k^{2} \mu}\right\rangle\right\}=\exp \left\{-\left\langle\Psi_{k} f, \mu\right\rangle\right\}
$$

where

$$
\left(\Psi_{k} f\right)(x)=\Psi_{k}[f(x)]
$$

with

$$
\Psi_{k}(t)=2 k^{2}[1-\exp \{-t / k\}] .
$$

Hence $\Psi_{k}$ defined by (2.8)-(2.9) belongs to $\mathscr{A}$ for each $k$.

\section{CONSTRUCTION OF SUPERPROCESSES}

3.1. Suppose that a mapping $V_{u}^{r}$ from $B^{+}$to $B^{+}$is given for every $r<u \in \Delta$. Put $V_{u}^{r}=0$ for $r \geq u$. We say that $V=\left\{V_{u}^{r}\right\}$ is an $\mathscr{A}$-semigroup if:

3.1.A. $V_{u}^{r}=V_{t}^{r} V_{u}^{t}$ for every $r<t<u \in \Delta$.

3.1.B. For every $f \in B^{+}, u \in \Delta, V_{u}^{r} f(x)$ is $\mathscr{B}_{\Delta} \times \mathscr{B}$-measurable in $r, x$.

3.1.C. $V_{u}^{r} \in \mathscr{A}$ for all $r<u \in \Delta$.

It follows from 2.4.C that to every $\mathscr{A}$-semigroup $V$ there corresponds a Markov transition function $q$ in $\left(\mathscr{M}, \mathscr{B}_{\mathscr{M}}\right)$ which is connected with $V$ by (1.3). By 2.4.D, the $U$-limit of $\mathscr{A}$-semigroups is also an $\mathscr{A}$-semigroup.

3.2. Theorem 3.1. Let $p$ be a Markov transition function subject to condition 1.1.A and let $T_{u}^{r}, r<u \in \Delta$, be the corresponding Markov semigroup. Then there exists a unique $\mathscr{A}$-semigroup $V$ which satisfies the equation (1.2). It is the $U$-limit of $\mathscr{A}$-semigroups $W(k)$ determined by the equation

$$
W_{u}^{r} f=-\int T_{s}^{r} \Phi_{k} W_{u}^{s} f d s+T_{u}^{r} f, \quad f \in B^{+}, r<u \in \Delta,
$$

where

$$
\Phi_{k} f=2 k f-\Psi_{k} f
$$

and $\Psi_{k}$ is given by (2.8)-(2.9). The operators $V_{u}^{r}$ are contractions.

3.3. Proof of Theorem 3.1 is based on two lemmas.

Lemma 3.1 (Gronwall's inequality). Suppose that a bounded positive function satisfies the condition

$$
a(r) \leq \alpha+\beta \int_{r}^{u} a(s) d s \quad \text { for } r \in[t, u]
$$

where $\alpha$ and $\beta$ are positive constants. Then

$$
a(r) \leq \alpha e^{\beta(u-r)} \quad \text { for } r \in[t, u]
$$


Proof. If $a(r) \leq b$ on $[t, u]$, then we establish by induction that

$$
a(r) \leq \sum_{i=0}^{n-1} \frac{\alpha \beta^{i}(u-r)^{i}}{i !}+\frac{b \beta^{n}(u-r)^{n}}{n !} \text { for } n=1,2, \ldots
$$

Passing to the limit as $n \rightarrow \infty$, we get (3.10).

Lemma 3.2. Fix $a k>0$ and put

$$
\begin{gathered}
U_{u}^{r}(1)=S_{u}^{r}=e^{-2 k(u-r)} T_{u}^{r}, \\
U_{u}^{r}(n+1)=\int S_{s}^{r} \Psi_{k} U_{u}^{s}(n) d s+S_{u}^{r} \text { for } n=1,2, \ldots
\end{gathered}
$$

The $U$-limit $W$ of $U(n)$ exists. It is an $\mathscr{A}$-semigroup and it satisfies (3.1). Proof. The operator $S_{u}^{r}$ belongs to $\mathscr{A}$ [the corresponding $q(\mu, d \nu)$ is the unit mass at the point $\left.\mu_{u}(\cdot)=\int \mu(d x) p(r, x ; u, \cdot) e^{-2 k(u-r)}\right]$. By 2.4.A-C and (2.5), $U_{u}^{r}(n) \in \mathscr{A}$ for all $n$. We prove by induction that

$$
\left\|U_{n}^{r}(n+1) f-U_{n}^{r}(n) f\right\| \leq(2 k)^{n}(u-r)^{n}(n !)^{-1}\|f\| .
$$

Therefore the $U$-limit $W$ of $U(n)$ exists and satisfies the equation

$$
W_{u}^{r}=\int S_{s}^{r} \Psi_{k} W_{u}^{s} d s+S_{u}^{r}, \quad r<u \in \Delta .
$$

An elementary computation shows that the function

$$
\varphi_{u}^{r}=\int_{r}^{u} S_{s}^{r} h_{u}^{s} d s+S_{u}^{r} f, \quad r<u \in \Delta
$$

satisfies the equation

$$
\varphi_{u}^{r}+2 k \int_{r}^{u} T_{s}^{r} \varphi_{u}^{s} d s=\int_{r}^{u} T_{s}^{r} h_{u}^{s} d s+T_{u}^{r} f, \quad r<u \in \Delta .
$$

We get (3.1) by applying (3.8) to $\varphi_{u}^{r}=W_{u}^{r} f, h_{u}^{s}=\Psi_{k} W_{u}^{s} f$ (for which (3.7) is equivalent to (3.6)).

To prove that $W_{u}^{r}=W_{s}^{r} W_{u}^{s}$ for $r<s<u \in \Delta$, we note that $h_{r}=W_{u}^{r} f$ and $\tilde{h}_{r}=W_{t}^{r} h_{t}$ satisfy the relation

$$
\left\|h_{r}-\tilde{h}_{r}\right\| \leq 2 k \int_{r}^{t}\left\|h_{s}-\tilde{h}_{s}\right\| d s,
$$

and $h_{r}=\tilde{h}_{r}$ by Lemma 3.1 .

\subsection{Proof of Theorem 3.1. Note that}

$$
\Phi_{k}(t)=t^{2}+\mathscr{W}^{k}(t)
$$

where

$$
\left|\mathscr{W}^{k}(t)\right| \leq t^{3} / k
$$

and therefore

$$
\left|\Phi^{l}\left(t_{2}\right)-\Phi^{k}\left(t_{1}\right)\right| \leq\left|t_{2}^{2}-t_{1}^{2}\right|+\left|\mathscr{W}^{l}\left(t_{2}\right)\right|+\left|\mathscr{W}^{k}\left(t_{1}\right)\right| \leq 2 c\left|t_{2}-t_{1}\right|+c^{3} / k
$$

for all $k<l, 0 \leq t_{1}, t_{2} \leq c$. 
Set $f \in B_{c}^{+}$if $f \in B^{+}$and $\|f\| \leq c$. It follows from (3.1) and (3.11) that, for all $l>k, t<u \in \Delta, f \in B_{c}^{+}$,

$$
a(r)=\left\|W_{u}^{r}(l) f-W_{u}^{r}(k) f\right\|
$$

satisfies (3.2) with $\alpha=c^{3} / k, \beta=2 c$ and, by Lemma 3.1,

$$
\left\|W_{u}^{r}(l) f-W_{u}^{r}(k) f\right\| \leq c^{3} e^{2 c(u-r)} / k .
$$

This implies that the $U$-limit $V_{u}^{r}(f)$ of $W_{u}^{r}(k)$ exists and

$$
\left\|V_{u}^{r} f-W_{u}^{r}(k) f\right\| \leq c^{3} e^{2 c(u-r)} / k
$$

for all $k=1,2, \ldots, r<u \in \Delta, f \in B_{c}^{+}$. Clearly $V_{u}^{r}$ is a semigroup. By 2.4.D it is an $\mathscr{A}$-semigroup. Passing to the limit in (3.1), we get (1.2). It follows from (1.2) that, for every $f \in B^{+}, 0 \leq V_{u}^{r} f \leq T_{u}^{r} f$ and therefore $\left\|V_{u}^{r} f\right\| \leq\|f\|$.

3.6. To prove that the series (1.9) is dominated by the numerical series (1.11), we note that, by (1.6),

$$
\|\varphi * \psi\| \leq|\Delta|\|\varphi\|\|\psi\|
$$

Therefore

$$
\left\|\varphi^{n *}\right\| \leq B_{n}|\Delta|^{n-1}\|\varphi\|^{n}
$$

where

$$
B_{1}=1, \quad B_{n}=B_{1} B_{n-1}+B_{2} B_{n-2}+\cdots+B_{n-1} B_{1} .
$$

Let $f(\theta)=\frac{1}{2}-\frac{1}{2} \sqrt{1-4 \theta}=C_{1} \theta+\cdots+C_{n} \theta^{n}+\cdots,|\theta|<\frac{1}{4}$. Since $f=\theta+f^{2}$ and $f(0)=0$, the equations (3.14) hold for $C_{n}$ and $C_{n}=B_{n}$. We note that $\lim \sup \left|C_{n}\right|^{1 / n}=4$ and, by (3.13) lim sup $\left\|\varphi^{n *}\right\|^{1 / n} \leq 4|\Delta|\|\varphi\|$ which implies (1.12).

\section{Stochastic Integrals}

4.1. Here we prove results on stochastic integrals stated in $\S \S 1.6$ and 1.7. In $\S 4$ we set $T_{s}^{s} f=f$ for all $f$.

Lemma 4.1. For every $h \in \mathscr{R}$, there exists a sequence of broken exit laws $h_{k}$ such that $\left\|h_{k}\right\| \leq\|h\|$ and $h_{k} \rightarrow h$ pointwise.

Proof. Fix a finite subset $\Gamma=\left\{t_{0}<t_{1}<\cdots<t_{n}\right\}$ of $\Delta$ which contains the right end $b$ of $\Delta$ if that belongs to $\Delta$. Let $\Delta_{0}=\Delta_{\leq t_{0}}, \Delta_{1}=\left(t_{0}, t_{1}\right], \ldots$, $\Delta_{n}=\left(t_{n-1}, t_{n}\right], \Delta_{n+1}=\Delta_{>t_{n}}$. (If $b \in \Delta$, then $b=t_{n}$ and $\Delta_{n+1}$ is empty.) Put

$$
\begin{gathered}
\beta(s)=t_{i}, \quad \text { for } s \in \Delta_{i}, i=0, \ldots, n ; \\
h_{\Gamma}^{s}= \begin{cases}T_{\beta(s)}^{s} h^{\beta(s)} & \text { for } s \in \Delta_{0} \cup \cdots \cup \Delta_{n}, \\
0 & \text { for } s \in \Delta_{n+1} .\end{cases}
\end{gathered}
$$

Obviously $h_{\Gamma} \in \mathscr{E}$ and $\left\|h_{\Gamma}\right\| \leq\|h\|$. 
We note that $T_{t}^{s} h^{t}(x)=\Pi_{s, x} h^{t}\left(\xi_{t}\right) \rightarrow \Pi_{s, x} h^{s}\left(\xi_{s}\right)=h^{s}(x)$ for all $s, x$ as $t \downarrow s$. Let $\Gamma$ run over a monotone sequence of sets with the union dense in $\Delta$. Then $\beta(s) \downarrow s$ and $h_{\Gamma}^{s}(x) \rightarrow h^{s}(x)$ for all $s \in \Delta, x \in E$.

Lemma 4.2. If $h$ is a bounded $\mathscr{C}$-measurable function, then there exists a sequence $h_{k} \in \mathscr{E}$ such that

$$
P \int_{r}^{u}\left\langle\left(h_{k}^{s}-h^{s}\right)^{2}, X_{s}\right\rangle d s \rightarrow 0
$$

for all $r<u \in \Delta$ and all Markov processes $\left(X_{t}, \mathscr{F}_{t}, P\right)$ with transition function $q$.

Proof. The set $H$ of all functions $h$ for which this statement is true is closed in $\mathscr{H}$. Therefore it is closed under the bounded convergence which implies the convergence in $\mathscr{H}$. Since $H$ contains 1 and the multiplicative family $\mathscr{R}$, it contains all bounded $\mathscr{C}$-measurable functions by the Multiplicative Systems Theorem.

Remark. For an arbitrary $h \in \mathscr{K}$, the formula

$$
h_{k}=(-k) \vee(k \wedge h)
$$

defines a sequence of bounded $\mathscr{C}$-measurable functions which converges to $h$ in $\mathscr{H}$. Therefore $\mathscr{E}$ is everywhere dense in $\mathscr{K}$.

4.2. First, we define the value $I_{u}^{r}(h)$ for $h \in \mathscr{E}$. Suppose that $h$ is an exit law on intervals $\Delta_{0}, \Delta_{1}, \ldots, \Delta_{n}, \Delta_{n+1}$ described in Lemma 4.1. We define $I_{u}^{r}=I_{u}^{r}(h)$ by the formula

$$
I_{u}^{r}=\left\langle h^{u}, X_{u}\right\rangle-\left\langle h^{r}, X_{r}\right\rangle \text { for } r, u \in \Delta_{i}, i=0,1, \ldots, n+1 .
$$

By (1.17), (1.19) and (1.25), formula (1.30) holds for $\tilde{h}=h$. Taking into account (1.27), we have

$$
P I_{u}^{r} I_{u}^{r^{\prime}} \rightarrow P \int_{t_{i-1}}^{u}\left\langle\left(h^{s}\right)^{2}, X_{s}\right\rangle d s \quad \text { as } r, r^{\prime} \downarrow t_{i-1} .
$$

Therefore there exists an $L^{2}(P)$-limit

$$
I_{u}^{t_{i-1}}=\lim _{r \downarrow t_{i-1}} I_{u}^{r}
$$

and (1.30), with $\tilde{h}=h$, is satisfied also for $r=t_{i-1}$. By (1.27), it holds for all $r<u \in \Delta$ if we put

$$
I_{u}^{r}=I_{t_{i}}^{r}+I_{t_{i+1}}^{t_{i}}+\cdots+I_{u}^{t_{j-1}} \text { for } r \in \Delta_{i}, u \in \Delta_{j}, r<u
$$

Clearly, $I(h)$ defined by $(4.4),(4.6)$ and (4.7) belongs to $\mathscr{L}_{f}$. Since $\mathscr{E}$ is a linear space and since $I(h+\tilde{h})=I(h)+i(\tilde{h}),(1.30)$ holds for all $h, \tilde{h} \in \mathscr{E}$, and $h \rightarrow I(h)$ is an isometry from $\mathscr{E}$ to $\mathscr{L}_{f}$. By remark at the end of $\S 4.1$, it can be continued, in a unique way, to an isometry from $\mathscr{K}$ to $\mathscr{L}_{f}$. 
To investigate the range of $I$, we put $\mathscr{Q}_{u}^{r}=\left\{Y: Y \in L_{u}^{r}, P\left(Y \mid \mathscr{F}_{r}\right)=0\right\}$. For every $r<u \in \Delta, I_{u}^{r}$ is an isometry from $\mathscr{H}_{u}^{r}=L^{2}\left([r, u] \times E, \mu_{u}^{r}\right)$ to $\mathscr{Q}_{u}^{r}$. Suppose that a $Y \in \mathscr{Q}_{u}^{r}$ is orthogonal to the range of $I_{u}^{r}$. Let $t \in[r, u], f \in B^{+}$ and let $h^{s}=T_{t}^{s} f$ for $s \in(r, t], h^{s}=0$ for $s \notin(r, t]$. We have

$$
I_{u}^{r}(h)=I_{t}^{r}(h)=\left\langle h^{t}, X_{t}\right\rangle-\left\langle h^{r}, X_{r}\right\rangle=\left\langle f, X_{t}\right\rangle-P\left\{\left\langle f, X_{t}\right\rangle \mid \mathscr{F}_{r}\right\}
$$

and therefore $0=P Y I_{u}^{r}(h)=P Y\left\langle f, X_{t}\right\rangle$. We conclude that $Y=0$ and thus $I_{u}^{r}\left(\mathscr{H}_{u}^{r}\right)=\mathscr{Q}_{u}^{r}$.

Now we prove that every $A \in \mathscr{L}_{f}$ is in the range of the mapping $I$. For every $r<u \in \Delta, A_{u}^{r}$ lies in $\mathscr{Q}_{u}^{r}$ and therefore $A_{u}^{r}=I_{u}^{r}(h)$ for some $h \in \mathscr{H}_{u}^{r}$. Our objective will be met if we show that the restriction $\tilde{h}$ of $h$ to an interval $[s, t] \subset[r, u]$ satisfies the equation $A_{t}^{s}=I_{t}^{s}(\tilde{h})$. This follows from the relation $P A_{u}^{r} I_{t}^{s}(g)=P A_{t}^{s} I_{t}^{s}(g)$ (cf. (1.27)) and formula (1.30).

4.3. To define a canonical version of $I(h)$ with properties described in $\S 1.7$, we use the concept of the medial limit due to Mokobodski. It is possible (see Dellacherie and Meyer (1983), $\S \S X .55-X .57)$ to associate with ever sequence $\left(a_{n}\right)$ of positive numbers an element of the extended half-line $[0,+\infty]$ denoted by $\lim \operatorname{med} a_{n}$ such that:

4.3.A. $\lim \inf a_{n} \leq \lim \operatorname{med} a_{n} \leq \lim \sup a_{n}$.

4.3.B. If $Y_{n}(\omega)$ is a sequence of $\mathscr{F}$-measurable functions, then $Y(\omega)=$ lim med $Y_{n}(\omega)$ is measurable relative to the universal completion of $\mathscr{F}$.

For an arbitrary sequence of real numbers $\lim \operatorname{med} a_{n}=\lim \operatorname{med} a_{n}^{+}-$ $\lim \operatorname{med} a_{n}^{-}$if $\lim \operatorname{med}\left|a_{n}\right|<\infty$. Otherwise $\lim \operatorname{med} a_{n}$ remains undefined.

With this definition we have the following properties:

4.3.C. If $Y_{n} \rightarrow Y$ in measure $P$, then $\lim \operatorname{med}\left|Y_{n}\right|<\infty, P$-a.s. and $Y=$ $\lim \operatorname{med} Y_{n} P$-a.s.

4.3.D. If $Y_{n}$ are uniformly integrable with respect to $P$, then $\lim$ med $\left|Y_{n}\right|<$ $\infty \quad P$-a.s. and $\lim \operatorname{med} \int Y_{n} d P=\int \lim \operatorname{med} Y_{n} d P$.

It follows from 4.3.C and $D$ that:

4.3.E. If $Y_{n} \rightarrow Y$ in $L^{2}(P)$, then $\lim \operatorname{med}\left|Y_{n}\right|<\infty \quad P$-a.s. and $\lim \operatorname{med} Y_{n}$ $=Y \quad P$-a.s.

If $h \in \mathscr{E}$, then we define $I_{u}^{r}=I_{u}^{r}(h)$ by (4.4) and (4.7) with (4.6) replaced by the formula

$$
I_{u}^{t_{i-1}}= \begin{cases}\lim _{r \downarrow t_{i-1}} \operatorname{med} I_{u}^{r} & \text { if the limit is defined } \\ 0 & \text { otherwise. }\end{cases}
$$

For every $P \in \mathscr{P}_{s}$ and every $r<u \in \Delta_{\geq s}$, the limits (4.7) and (4.8) coincide $P$-a.s. by 4.3 .E.

If $h$ is a bounded $\mathscr{C}$-measurable function, then we put ,

$$
I_{u}^{r}(h)= \begin{cases}\lim \operatorname{med} I_{u}^{r}\left(h_{k}\right) & \text { if the limit is defined } \\ 0 & \text { otherwise }\end{cases}
$$


where $h_{k} \in \mathscr{E}$ is the sequence in Lemma 4.2 . For an arbitrary $\mathscr{C}$-measurable function $h$, we again use formula (4.9) with $h_{k}$ defined by (4.3). By 4.3.E, $I(h)$ is a fair linear additive functional of the family $\mathscr{X}$.

4.4. If 1.7.A holds, then the canonical version of $I_{u}^{s}(h)$ is right continuous in $u$ on $\Delta_{\geq s} P$-a.s. for all $s \in \Delta$ and $P \in \mathscr{P}_{s}$. Indeed, fix $s \in \Delta, P \in \mathscr{P}_{s}$. First we note that if $h$ is an exit law on $(a, u] \subset \Delta_{\geq s}$, then, by (1.25), (1.15) and 1.7.A

$$
\left\langle h^{r}, X_{r}\right\rangle=\left\langle T_{u}^{r} h^{u}, X_{r}\right\rangle=P_{r, X_{r}}\left\langle h^{u}, X_{u}\right\rangle
$$

is right continuous on $(a, u)$ and tends to a limit as $r \downarrow a$, for $P$-almost all $\omega$.

If $h$ is a bounded $\mathscr{C}$-measurable function and if $h_{k}$ are the elements of $\mathscr{E}$ in Lemma 4.2, then

$$
P\left[I_{u}^{s}\left(h_{k}\right)-I_{u}^{s}(h)\right]^{2} \rightarrow 0 \text { for all } u \in \Delta_{\geq s},
$$

and the right continuity of $I_{u}^{s}(h)$ in $u$ follows, in a routine way, from Kolmogorov's inequality for martingales. The same arguments are applicable to an arbitrary $\mathscr{C}$-measurable $h$.

4.5. Looking through the construction of the stochastic integral, we establish the following result [cf. Walsh (1986) and Dynkin (1987)]. Suppose that the integrand $h$ depends on a parameter $\gamma$ with values in a measurable space $\left(\Gamma, \mathscr{B}_{\Gamma}\right)$. If $h_{\gamma}^{s}(x)$ is jointly measurable in $\gamma, s, x$ and if $h_{\gamma} \in \mathscr{K}$ for every $\gamma$, then one can choose a $\mathscr{B}_{\Gamma}$-measurable version of $I_{u}^{r}\left(h_{\gamma}\right)$. Moreover, if $\nu$ is a measure on $\left(\Gamma, \mathscr{B}_{\Gamma}\right)$ such that $h=\int h_{\gamma} \nu(d \gamma) \in \mathscr{K}$, then $I_{u}^{r}(h)=\int I_{u}^{r}\left(h_{\gamma}\right) \nu(d \gamma)$.

\section{Positive Linear additive functionals}

5.1. Lemma 5.1. Let $h$ be an exit rule. Then $h$ is $\mathscr{B}_{\Delta} \times \mathscr{B}-$ measurable. Under the assumption 1.7.A, for every $s \in \Delta, P \in \mathscr{P}_{s}$, the function $\left\langle h^{t}, X_{t}\right\rangle$ is right continuous in $t$ on $\Delta_{\geq s} P$-a.s.

Proof. Let $h_{\Gamma}^{s}$ be defined by formula (4.1) with $\Delta_{0}=\Delta_{<t_{0}}, \Delta_{1}=\left[t_{0}, t_{1}\right), \ldots$, $\Delta_{n}=\left[t_{n-1}, t_{n}\right), \Delta_{n+1}=\Delta_{\geq t_{n}}$. If $h$ is an exit rule, then

$$
h_{\Gamma} \leq h \text { and } h_{\Gamma} \uparrow h
$$

(same passage to the limit as in Lemma 4.1). Since $h_{\Gamma}$ is $\mathscr{B}_{\Delta} \times \mathscr{B}$-measurable by 1.1.A, $h$ also is $\mathscr{B}_{\Delta} \times \mathscr{B}$-measurable.

By Meyer (1968) [Chapter VI, Theorem 16], if positive supermartingales $Y_{n}(t)$ are right continuous a.s., and if $Y_{n}(t) \uparrow Y(t)$ for all $t$, then $Y(t)$ is also right continuous a.s. Therefore Lemma 5.1 will be proved if we show $\left\langle h_{\Gamma}^{t}, X_{t}\right\rangle$ is a right continuous supermartingale. This follows from the relation

$$
h_{\Gamma}^{s}=\sum_{i=1}^{n-1} T_{t_{i}}^{s} f_{i}+T_{t_{n}}^{s} h^{t_{n}}
$$

where

$$
f_{i}=h^{t_{i}}-T_{t_{i+1}}^{t_{i}} h^{t_{i+1}} \geq 0, \quad \text { and } \quad T_{t}^{s}=0 \text { for } s \geq t .
$$


5.2. Lemma 5.2. Denote by $\mathscr{V}_{s}$ the minimal linear space which contains $\left\langle f, X_{t}\right\rangle$ for all $f \in B^{+}, t \in \Delta_{\geq s}$. If $Y_{n} \in \mathscr{V}_{s}$ for $n=1,2, \ldots$ and if

$$
P\left(Y-Y_{n}\right)^{2} \rightarrow 0 \text { for all } P \in \mathscr{P}_{s},
$$

then

$$
P_{s, \nu} Y=\int P_{s, \delta_{x}} Y \nu(d x)
$$

for every $\nu \in \mathscr{M}$.

Proof. The relation (5.2) holds for $Y_{n} \in \mathscr{V}_{s}$ by (1.15). Since measures $P_{s, \nu}$ and $Q=\int P_{s, \delta_{x}} \nu(d x)$, belongs to $\mathscr{P}_{s}$, we have

$$
\lim P_{s, \nu}\left(Y-Y_{n}\right)^{2}=\lim Q\left(Y-Y_{n}\right)^{2}=0
$$

and (5.2) holds for $Y$.

5.3. If $h$ is the characteristic of $A \in \mathscr{N}$, then, by Lemmas 4.2 and 5.2,

$$
P_{t, \nu} A\left(\Delta_{>t}\right)=\left\langle h^{t}, \nu\right\rangle
$$

and

$$
T_{t}^{r} h^{t}(x)=P_{r, \delta_{x}}\left\langle h^{t}, X_{t}\right\rangle=P_{r, \delta_{x}} P_{t, X_{t}} A\left(\Delta_{>t}\right)=P_{r, \delta_{x}} A\left(\Delta_{>t}\right) .
$$

Hence $h$ is an exit rule.

Let $g$ be an exit law and let $0 \leq g \leq h$. Then by (5.4),

$$
0 \leq g^{r}(x)=T_{t}^{r} g^{t}(x) \leq P_{r, \delta_{r}} A\left(\Delta_{>t}\right) .
$$

Denote by $b$ the right end of $\Delta$. Put $t=b$ if $b \in \Delta$ and let $t \uparrow b$ if $b \notin \Delta$. We get that $g^{r}(x)=0$. Hence $h$ is a pure exit rule.

5.4. Theorem 5.1. Suppose that 1.7.A, B are satisfied and let $h$ be a bounded pure exit rule. The canonical version of the stochastic integral can be specified in such a way that

$$
A_{u}^{r}=\int_{r}^{u} h^{s} d Z_{s}+\left\langle h^{r}, X_{r}\right\rangle-\left\langle h^{u}, X_{u}\right\rangle
$$

is a positive linear additive functional of $\mathscr{X}$. Moreover the characteristic of $A$ is $h$ and every positive linear additive functional $A$ with the characteristic $h$ is equivalent to $A$.

Proof. By Theorem 7.2 (which will be proved without any reference to $\S 5$ ), there exists a canonical version of $I(h)$ such that $I_{u}^{r}(h)$ is continuous in $u$ in $\Delta_{\geq r} P$-a.s. for every $r \in \Delta, P \in \mathscr{P}_{r}$.

$1^{\circ}$. We claim that

$$
A_{u}^{r}=I_{u}^{r}(h)+\left\langle h^{r}, X_{r}\right\rangle-\left\langle h^{u}, X_{u}\right\rangle \geq 0 \quad P \text {-a.s. }
$$

for all $P \in \mathscr{P}_{r}$. Define $h_{\Gamma}$ by formula (4.1). Fix $r \in \Delta$ and $P \in \mathscr{P}_{r}$. By (5.1),

$$
I_{u}^{r}\left(h_{\Gamma}\right) \rightarrow I_{u}^{r}(h) \text { in } L^{2}(P) .
$$


Hence $A_{u}^{r}$ is the $L^{2}$-limit of

$$
A_{u}^{r}(\Gamma)=I_{u}^{r}(\Gamma)+\left\langle h^{r}, X_{r}\right\rangle-\left\langle h^{u}, X_{u}\right\rangle .
$$

If $t \in \Delta_{i}, i \leq n$, then, by (1.29) and (5.1), $A_{t_{i}}^{t}(\Gamma)=\left\langle h^{t}-h_{\Gamma}^{t}, X_{t}\right\rangle$ is nonnegative. By 1.7.A and (4.6), $A_{t_{i}}^{t_{i-1}}(\Gamma) \geq 0 \quad P$-a.s. for $t_{i-1} \geq r$. Hence $A_{\beta(u)}^{r}(\Gamma) \geq 0 P$ a.s. for all $u \in \Delta_{\geq r}$. On the other hand, $A_{\beta(u)}^{u}(\Gamma)=\left\langle h^{u}-T_{\beta(u)}^{u} h^{\beta(u)}, X_{u}\right\rangle \rightarrow 0$ and (5.6) follows.

$2^{\circ}$. For every $r \in \Delta$, we denote $\Lambda_{r}$ the set which consists of $r$ and all rationals in $\Delta_{>r}$. Put

$$
\omega \in \Omega_{r} \quad \text { if } \infty>A_{u_{2}}^{u_{1}} \geq 0
$$

and

$$
A_{u_{2}}^{u_{1}}+A_{u_{3}}^{u_{2}}=A_{u_{3}}^{u_{1}} \quad \text { for all } u_{1}<u_{2}<u_{3} \in \Lambda_{r}
$$

and if $A_{u}^{r}$ is right continuous in $u$ on $\Delta_{\geq r}$. By Lemma 5.1, 1.5.B and (5.6), $P\left(\Omega \backslash \Omega_{r}\right)=0$ for all $P \in \mathscr{P}_{r}$. Put

$$
\widetilde{A}_{u}^{r}= \begin{cases}A_{u}^{r} & \text { if } \omega \in \Omega_{r}, \\ 0 & \text { if } \omega \notin \Omega_{r} .\end{cases}
$$

Obviously, $\tilde{A} \in \mathcal{N}$ and $\widetilde{I}_{u}^{r}(h)=\widetilde{A}_{u}^{r}+\left\langle h^{u}, X_{u}\right\rangle-\left\langle h^{r}, X_{r}\right\rangle$ is a continuous canonical version of the stochastic integral. By dropping tildes, we get (5.5).

$3^{\circ}$. By (5.5), (1.23) and (1.15),

$$
P_{r, \delta_{x}} A_{u}^{r}=h^{r}(x)-T_{u}^{r} h^{u}(x)
$$

and therefore

$$
P_{r, \delta_{x}} A\left(\Delta_{>r}\right)=h^{r}(x)-g^{r}(x)
$$

where $g^{r}(x)=T_{b}^{r} h^{b}(x)$ if the right end $b$ of $\Delta$ belongs to $\Delta$ or $g^{r}(x)=$ $\lim _{u \uparrow b} T_{u}^{r} h^{u}(x)$ if $b \notin \Delta$. In both cases $g$ is an exit law such that $0 \leq g \leq h$ and, since $h$ is pure, $g=0$. Thus the characteristic of $A$ is equal to $h$.

$4^{\circ}$. Suppose that $A \in \mathscr{N}$ has a bounded characteristic $h$. Fix $s \in \Delta$ and $P \in \mathscr{P}_{s}$. By (5.3), for $r<u \in \Delta_{\geq s}$,

$$
P\left\{A_{u}^{r} \mid \mathscr{F}[s, r]\right\}=P_{r, X_{r}} A_{u}^{r}=\left\langle h^{r}, X_{r}\right\rangle-\left\langle h^{u}, X_{u}\right\rangle \quad P \text {-a.s. }
$$

and therefore

$$
B_{u}^{r}=A_{u}^{r}-\left\langle h^{r}, X_{r}\right\rangle+\left\langle h^{u}, X_{u}\right\rangle
$$

is a fair linear additive functional of $\left(X_{t}, \mathscr{F}[s, t], P\right)$. We have proved in $\S 4.2$ that there exists an $f \in \mathscr{H}(P)$ such that $B=I(f) \quad P$-a.s., and therefore

$$
A_{u}^{r}=I_{u}^{r}(f)+\left\langle h^{r}, X_{r}\right\rangle-\left\langle h^{u}, X_{u}\right\rangle .
$$

As we know,

$$
C_{u}^{r}=I_{u}^{r}(h)+\left\langle h^{r}, X_{r}\right\rangle-\left\langle h^{u}, X_{u}\right\rangle
$$

is another positive linear additive functional. We note that $A_{u}^{r}-C_{u}^{r}=I_{u}^{r}(h-f)$, $u \in \Delta_{>r}$, is a continuous square-integrable martingale with bounded variation. 
For such a martingale the quadratic variation vanishes and therefore $A=C$ $P$-a.s.

\section{LAPLACE TRANSFORMS OF STOCHASTIC INTEGRALS}

6.1. Theorem 6.1. Formulae (1.39), (1.40), and (1.41) hold for every $\mathscr{C}$ measurable function $h$ such that

$$
4\|h\||\Delta|<1 .
$$

Proof. $1^{\circ}$. It is easy to see that (1.40) and (1.41) are equivalent if $h$ satisfies (6.1). Fix $\rho<(4|\delta|)^{-1}$ and denote by $H$ the class of all functions $h$ such that (1.39) holds for

$$
h_{\rho}=(-\rho) \vee(\rho \wedge h) .
$$

Put $B_{\rho}=\{h:\|h\| \leq \rho\}$. It is sufficient to prove that $H$ is closed under bounded convergence and that it contains all broken exit laws $h \in B_{\rho}$. Indeed, then, by Lemma 4.1, $H \supset \mathscr{R} \cap B_{\rho}$ which implies that $H \supset \mathscr{R}$, and $H$ contains all $\mathscr{C}$-measurable functions by the Modified Multiplicative Systems Theorem.

$2^{\circ}$. Let $h(n) \in H$ converge pointwise to $h$ and let $w(n), w$ correspond to $h(n)_{\rho}, h_{\rho}$ by formula (1.40). By (1.12), w(n) $\rightarrow w$ pointwise. By 1.6.B, $A_{u}^{r}(n)=I_{n}^{r}\left(h(n)_{\rho}\right)$ converge to $A_{u}^{r}=I_{u}^{r}\left(h_{\rho}\right)$ in $L^{2}\left(P_{r, \mu}\right)$. For some subsequence $n_{i}, A_{u}^{r}\left(n_{i}\right) \rightarrow A_{u}^{r} P_{r, \mu}$-a.s. Changing notations, we can assume that $A_{u}^{r}(n) \rightarrow A_{u}^{r}$ $P$-a.s. By Fatou's lemma,

$$
\begin{aligned}
P_{r, \mu} \exp A_{u}^{r} & \leq \liminf P_{r, \mu} \exp A_{u}^{r}(n) \\
& \leq \lim \exp \left\langle w_{u}^{r}(n), \mu\right\rangle=\exp \left\langle w_{u}^{r}, \mu\right\rangle<\infty
\end{aligned}
$$

Choose $k>1$ such that $k \rho<(4|\Delta|)^{-1}$. We have

$$
\sup _{n} P_{r, \mu}\left\{\exp A_{u}^{r}(n)\right\}^{k}=\sup _{n} \exp \left\langle\tilde{w}_{u}^{r}, \mu\right\rangle
$$

where $\tilde{w}(n)$ corresponds to $k h(n)_{\rho}$ by formula (1.40). By (1.12), $\|\tilde{w}(n)\| \leq$ $c_{k \rho} /\left(1-\beta_{k \rho}\right)$ with $\beta_{k \rho}<1$. Therefore (see, e.g., Meyer (1968), Chapter 2 , Theorem 2) the random variables $\exp A_{u}^{r}(n)$ are uniformly integrable and $P_{r, \mu} \exp A_{u}^{r}(n) \rightarrow P_{r, \mu} \exp A_{u}^{r}$. Thus $h \in H$.

$3^{\circ}$. Formula (1.39) holds if $h^{s}$ is an exit law on $\left(r_{0}, u\right]$. Indeed, in this case $h^{s}=T_{u}^{s} h^{u}$ for $s \in\left(r_{0}, u\right]$ and $A_{u}^{r}=\left\langle h^{u}, X_{u}\right\rangle-\left\langle h^{r}, X_{r}\right\rangle$. Hence

$$
F^{r}=\exp \left\{-\left\langle h^{r}, \mu\right\rangle\right\} P_{r, \mu} \exp \left\langle h^{u}, X_{u}\right\rangle
$$

and (1.39) follows from (1.13).

To prove that $H$ contains all broken exit laws, it is sufficient to check that (1.39) with $w$ defined by (1.41) holds for every $r<u \in \Delta$ if, for some $t \in \Delta$, it holds for all $r<u \in \Delta_{\leq t}$ and for all $r<u \in \Delta_{\geq t}$.

If $t \in \Delta$, then (1.41) is equivalent to the system of two equations

$$
v^{r}-\int_{r}^{u} T_{s}^{r}\left[\left(v^{s}\right)^{2}\right] d s=h^{r}, \quad r \in[t, u) ;
$$




$$
v^{r}-\int_{r}^{t} T_{s}^{r}\left[\left(v^{s}\right)^{2}\right] d s=\tilde{h}^{r}, \quad r \in \Delta_{<t}
$$

where

$$
\tilde{h}^{r}=h^{r}+T_{t}^{r} g^{t}
$$

with

$$
\dot{g}^{t}=\int_{t}^{u} T_{s}^{r}\left[\left(v^{s}\right)^{2}\right] d s .
$$

If (1.39) holds on $\Delta_{\geq t}$, then, for $r \in \Delta_{\leq t}, u \in \Delta_{\geq t}$,

$$
P_{r, \mu_{r}} \exp A_{u}^{r}=P_{r, \mu_{r}}\left[\exp A_{t}^{r} P_{t, X_{t}} \exp A_{u}^{t}\right]=P_{r, \mu_{r}} \exp \left\langle A_{t}^{r}+g^{t}, X_{t}\right\rangle
$$

with $g$ given by (6.7). By (1.29),

Therefore

$$
\left\langle g^{t}, X_{t}\right\rangle=\int_{r}^{t} T_{t}^{s} g^{t} d Z_{s}+\left\langle T_{t}^{r} g^{t}, X_{r}\right\rangle
$$

$$
P_{r, \mu} \exp A_{u}^{r}=P_{r, \mu} \exp \left\{\int_{r}^{t} \tilde{h}^{s} d Z_{s}+\left\langle T_{t}^{r} g^{t}, X_{r}\right\rangle\right\} .
$$

If (1.39) holds on $\Delta_{\geq t}$, then the right side is equal to

$$
\exp \left\{\left\langle T_{t}^{r} g^{t}+v^{r}-\tilde{h}^{r}, \mu\right\rangle\right\}=\exp \left\langle v^{r}-h^{r}, \mu\right\rangle
$$

which proves that (1.39) holds on $\Delta$.

6.2. Theorem 6.2. Let $\|h\|<\infty$. Put

$$
\beta(t)=\sup _{r \in[t, u)}\left\|v^{r}\right\|
$$

where $v^{r}$ is the solution of the equation (1.41). If

$$
r_{1}=\inf \{t: \beta(t)<\infty\}
$$

and if $r_{0}$ is defined by (1.42), then $r_{1}=r_{0}$, and (1.39) holds on $\left(r_{0}, u\right]$.

Proof. $1^{\circ}$. Suppose that (1.39) holds on $[t, u)$ and let $\left\|v^{t}\right\|=\beta<\infty$. By Theorem 6.1,

$$
P_{t, \mu_{r}} \exp \int_{r}^{t} \tilde{h}^{s} d Z_{s}=\exp \left\langle\tilde{h}^{r}-v^{r}, \mu_{r}\right\rangle \quad \text { for } r \in[t-\varepsilon, t)
$$

where $\tilde{h}$ is given by $(6.6)$ and $\varepsilon<(4\|\tilde{h}\|)^{-1}<[4\|h\|+(u-t) \beta]^{-1}$. By the argument in the proof of Theorem $6.1\left(\right.$ step $\left.3^{\circ}\right)(1.39)$ holds on $[t-\varepsilon, u)$.

$2^{\circ}$. Suppose that $\beta(t)<\infty$. Choose an $\varepsilon$ as in $1^{\circ}$ and consider a partition $t=t_{0}<t_{1}<\cdots<t_{n}=u$ such that $t_{i}-t_{i-1}<\varepsilon$ for $i=1, \ldots, n$. By induction, using $1^{\circ}$ and Theorem 6.1, we establish that (1.39) holds on $[t, u)$, and therefore it holds on $\left(r_{1}, u\right)$. Since

$$
\log P_{r, \delta_{x}} \exp \left\{\int_{r}^{u} h^{s} d Z_{s}\right\}=w_{u}^{r}(x)=v^{r}(x)-h^{r}(x) \quad \text { for } r>r_{1},
$$

we conclude that $r_{0} \leq r_{1}$. 
$3^{\circ}$. Suppose $r_{0}<r_{1}$. Then, by (6.10), $\beta=\beta\left(r_{1}\right)<\infty$. For every $t>r_{1}$, (1.39) holds on $[t, u)$. If $\varepsilon<(4\|h\|+(u-r) \beta)^{-1}$, then by $1^{\circ}$, formula (1.39) holds on $[t-\varepsilon / 2, u)$. By taking $t=r_{1}+\varepsilon / 2$, we conclude that (1.39) is satisfied on $\left[r_{1}-\varepsilon, u\right)$. The contradiction with the definition of $r_{1}$ shows that $r_{0}=r_{1}$.

\section{EXPONENTIAL MARTINGALE AND ITS APPLICATIONS}

7.1. Theorem 7.1. Let $A$ and $B$ be defined by (1.45) with a bounded $\mathscr{C}$ measurable function $h$ and let

$$
N_{u}^{r}(\alpha)=\alpha A_{u}^{r}-\alpha^{2} B_{u}^{r} / 2, \quad r<u \in \Delta .
$$

Then for all sufficiently small $\alpha$,

$$
P_{r, \mu} \exp N_{u}^{r}(\alpha)=1
$$

Proof. Put $\varphi_{t}^{s}=\alpha h^{s} 1_{s \leq t}$. Since

$$
f^{t}= \begin{cases}T_{s}^{t}\left[\left(h^{s}\right)^{2}\right] & \text { for } t \in(r, s), \\ \left(h^{s}\right)^{2} & \text { for } t=s\end{cases}
$$

is an exit law on $(r, s], 1.6 . \mathrm{A}$ implies that

$$
\left\langle\left(h^{s}\right)^{2}, X_{s}\right\rangle=\int_{r}^{s} T_{s}^{t}\left[\left(h^{s}\right)^{2}\right] d Z_{t}+\left\langle T_{s}^{r}\left[\left(h^{s}\right)^{2}\right], X_{r}\right\rangle .
$$

Therefore

$$
\begin{aligned}
\alpha^{2} \int_{r}^{u}\left\langle\left(h^{s}\right)^{2}, X_{s}\right\rangle d s & =\alpha^{2} \int_{r}^{u} d Z_{t} \int_{t}^{u} d s T_{s}^{t}\left[\left(h^{s}\right)^{2}\right]+\alpha^{2} \int_{r}^{u} d s\left\langle T_{s}^{r}\left[\left(h^{s}\right)^{2}\right], X_{r}\right\rangle \\
& =\int_{r}^{u} d Z_{t}(\varphi * \varphi)_{u}^{t}+\left\langle(\varphi * \varphi)_{u}^{r}, X_{r}\right\rangle
\end{aligned}
$$

and

$$
N_{u}^{r}(\alpha)=\int_{r}^{u}\left[\varphi_{u}^{s}-(\varphi * \varphi)_{u}^{s}\right] d Z_{s}-\left\langle(\varphi * \varphi)_{u}^{r}, X_{r}\right\rangle .
$$

By Theorem 6.1, for sufficiently small $\alpha$,

$$
P_{r, \mu} \exp N_{u}^{r}(\alpha)=\exp \left[-\left\langle(\varphi * \varphi)_{u}^{r}, \mu\right\rangle+\left\langle w_{u}^{r}, \mu\right\rangle\right]
$$

where

$$
v_{u}^{r}=\varphi_{u}^{r}-(\varphi * \varphi)_{u}^{r}+w_{u}^{r}
$$

is the unique solution of the equation

$$
v-v * v=\varphi-\varphi * \varphi .
$$

Hence $v=\varphi$ and $w=\varphi * \varphi$. Formula (7.2) follows from (7.3).

7.2. Remark 1. Formula $(7.2)$ implies that $\left(\exp \left\{N_{u}^{r}(\alpha)\right\}, \mathscr{F}_{u}, P_{r, \mu}\right)_{u \in \Delta \geq r}$ is a martingale. We call it the exponential martingale. 
Remark 2. The coefficient at $\alpha^{2}$ in the left side of (7.2) is equal to

$$
\frac{1}{2} P_{r, \mu}\left(A_{u}^{r}\right)^{2}-\frac{1}{2} P_{r, \mu} B_{u}^{r} \text {. }
$$

Since

$$
P\left\{\left(A_{u}^{r}\right)^{2} \mid \mathscr{F}_{r}\right\}=P_{r, X_{r}}\left(A_{u}^{r}\right)^{2}, \quad P\left\{B_{u}^{r} \mid \mathscr{F}_{r}\right\}=P_{r, X_{r}} B_{u}^{r},
$$

we get (1.46).

7.3. Theorem 7.2. Let $I_{u}^{r}(h)$ be the canonical right continuous version of the stochastic integral constructed in $\S 4$.4. Then for every $s \in \Delta, P \in \mathscr{P}_{s}, I_{u}^{s}(h)$ is continuous on $\Delta_{\geq s}$ P-a.s.

Proof. Put $A(r, u]=A_{u}^{s}-A_{r}^{s}, B(r, u]=B_{u}^{r}$ for all $s \leq r<u$. Note that, by 1.5.B, $A(r, u]=A_{u}^{r} P$-a.s.

By evaluating the coefficient at $\alpha^{4}$, in (7.2), we get

$$
P\left\{\frac{1}{24}\left(A_{u}^{r}\right)^{4}-\frac{1}{4} B_{u}^{r}\left(A_{u}^{r}\right)^{2}+\frac{1}{8}\left(B_{u}^{r}\right)^{2} \mid \mathscr{F}_{r}\right\}=0 \quad \text { for every } r<u \in \Delta .
$$

For any $\Gamma=\left\{t_{0}<t_{1}<\cdots<t_{n}\right\} \in \Delta$, we put

$$
S_{A}^{k}=\sum_{i=1}^{n} A\left(t_{i-1}, t_{i}\right]^{k}, \quad S_{B}^{k}=\sum_{i=1}^{n} B\left(t_{i-1}, t_{i}\right]^{k} .
$$

By the Schwarz inequality,

$$
P \sum_{i=1}^{n} B\left(t_{i-1}, t_{i}\right] A\left(t_{i-1}, t_{i}\right]^{2} \leq\left(P\left(S_{B}^{2}\right)\right)^{1 / 2}\left(P\left(S_{A}^{4}\right)\right)^{\mathrm{i} / 2}
$$

and, by (7.5),

which implies

$$
P\left(S_{A}^{4}\right) \leq 6\left(P\left(S_{B}^{2}\right)\right)^{1 / 2}\left(P\left(S_{A}^{4}\right)\right)^{1 / 2}
$$

$$
P\left(S_{A}^{4}\right) \leq 36 P\left(S_{B}^{2}\right) .
$$

By (1.15) and (1.16), for $s \leq r<t \leq u$,

$$
\begin{aligned}
P\left\langle f^{r}, X_{r}\right\rangle\left\langle f^{t}, X_{t}\right\rangle & =P\left[\left\langle f^{r}, X_{r}\right\rangle P_{r, X_{r}}\left\langle f^{t}, X_{t}\right\rangle\right] \\
& \leq\|f\|^{2} P\left\langle 1, X_{r}\right\rangle^{2} \leq C\|f\|^{2},
\end{aligned}
$$

where $C=\langle 1, \mu\rangle^{2}+2\langle 1, \mu\rangle(u-s)$. Therefore

$$
\begin{aligned}
P\left\{B\left(t_{i-1}, t_{i}\right]\right\}^{2} & =\int_{t_{i-1}}^{t_{i}} d r \int_{t_{i-1}}^{t_{i}} d t P\left\langle\left(h^{r}\right)^{2}, X_{r}\right\rangle\left\langle\left(h^{t}\right)^{2}, X_{t}\right\rangle \\
& \leq C\|h\|^{4}\left(t_{i}-t_{i-1}\right)^{2}
\end{aligned}
$$

and

$$
P S_{B}^{2} \leq C\|h\|^{4}(u-r) \max _{i}\left(t_{i}-t_{i-1}\right) .
$$


Consider an increasing sequence of sets $\Gamma_{n}$ with $\max _{i}\left(t_{i}-t_{i-1}\right) \rightarrow 0$. By (7.8) and (6.11), $P\left(S_{A}^{4}\right) \rightarrow 0$ and, by Fatou's lemma,

$$
P \liminf S_{A}^{4}=0 \text {. }
$$

Put

We have

$$
\alpha(r)=t_{i-1}, \quad \beta(r)=t_{i} \quad \text { for } r \in\left[t_{i-1}, t_{i}\right)
$$

Therefore

$$
A(\alpha(r), \beta(r)] \leq\left(S_{A}^{4}\right)^{1 / 4}
$$

$$
\sup _{r \in \Delta}\left|A_{r-}^{s}-A_{r}^{s}\right| \leq \liminf \left(S_{A}^{4}\right)^{1 / 4}=0 P \text {-a.s. }
$$

(The limits $A_{r-}^{s}$ exist for all $r \in \Delta_{\geq s} P$-a.s. because $\left(A_{r}^{s}, \mathscr{F}_{r}, P\right)$ is a right continuous martingale.)

\section{FELLER SUPERPROCESSES}

8.1. Now we assume that $E$ is a locally compact separable metric space and we denote by $E_{\partial}=E \cup \partial$ the one-point compactification of $E$. As usual, $C_{0}(E)$ means the space of continuous functions on $E$ which tend to 0 as $x \rightarrow \partial$. For every interval $\Delta$, we denote by $C_{0}(\Delta \times E)$ the space of real-valued functions $\varphi^{r}(x), r \in \Delta, x \in E$ such that:

(a) $\varphi^{r}(x) \rightarrow \varphi^{t}(y)$ as $r \downarrow t, x \rightarrow y$;

(b) $\varphi^{r}(x) \rightarrow 0$ as $r \downarrow t, x \rightarrow \partial$.

This is equivalent to the condition: $r \rightarrow \varphi^{r}$ is a strongly right-continuous mapping from $\Delta$ to $C_{0}(E)$.

8.2. Let $\mathscr{B}$ be the Borel $\sigma$-algebra in $E$ and let $V_{u}^{r}, r<u \in \Delta$, be a semigroup in the space $B^{+}$of bounded positive $\mathscr{B}$-measurable functions. We say that $V$ is a Feller semigroup if, for every positive $f \in C_{0}(E)$ and every $u \in \Delta$, the function $\varphi^{r}=V_{u}^{r} f$ belongs to $C_{0}(\Delta \times E)$. (We put $V_{u}^{r}=0$ for $r \geq u$.)

A transition function $p$ is $s$ called Feller if the corresponding semigroup (1.1) is Feller.

Suppose that $p$ is a Feller transition function and let $f^{r}=\varphi_{u}^{r}, g^{r}=\psi_{u}^{r}$ belong to $C_{0}(\Delta \times E)$. Then $h^{r}=(\varphi * \psi)_{u}^{r}$ also belongs to $C_{0}(\Delta \times E)$, and it follows from Lemma 3.3 that the semigroup $V_{u}^{r}$ is Feller.

8.3. Let $\mathscr{W}$ be the family of functions corresponding by (2.1) to $f \in C_{0}(E)$. These functions are continuous in the topology of $\mathscr{M}$ induced by $C_{0}(E)$. By the Stone-Weierstrass theorem, every function $F \in C_{0}(\mathscr{M})$ can be approximated uniformly by linear combinations of elements of $\mathscr{W}$, and we arrive at the following

Theorem 8.1. The supertransition function $q$ corresponding to a Feller transition function $p$ by (1.2), (1.3) is also Feller.

8.4. We say that a Feller semigroup $T_{u}^{r}$ is continuous if, for all $t \in \Delta$ and for all $f \in C_{0}(E),\left\|T_{u}^{r} f-f\right\| \rightarrow 0$ as $r, u \rightarrow t$. 
Theorem 8.2. If $T_{u}^{r}$ is a continuous Feller semigroup, then the corresponding supersemigroup $\mathscr{T}_{u}^{r}$ has the same property.

Proof. It is sufficient to show that

$$
\lim _{r, u \rightarrow t}\left\|\mathscr{T}_{u}^{r} F_{f}-F_{f}\right\|=0 \text { for all } f \in W
$$

where $F_{f}$ is given by (2.1). Since $\left|e^{-\alpha}-e^{-\beta}\right| \leq|\alpha-\beta|$ for $\alpha, \beta \geq 0$, we have

$$
\left\|\mathscr{T}_{u}^{r} F_{f}-F_{f}\right\| \leq\left|\left\langle V_{u}^{r} f-f, \mu\right\rangle\right| \leq\left\|V_{u}^{r} f-f\right\|\langle 1, \mu\rangle .
$$

By (1.2),

$$
\left\|V_{u}^{r} f-T_{u}^{r} f\right\|=\left\|\int_{r}^{u} T_{s}^{r}\left[\left(V_{u}^{s} f\right)^{2}\right] d s\right\| \leq\|f\|^{2}(u-r),
$$

and (8.1) follows from (8.2), (8.3) and continuity of $T_{u}^{r}$.

8.5. A Markov process $X_{t}, t \in \Delta$, with transition probabilities $P_{s, x}$ is called right if:

8.5.A. For all $r<u \in \Delta, f \in B^{+}, P \in \mathscr{P}_{r}$, the function $P_{s, X_{s}} f\left(X_{u}\right)$ is right continuous in $s$ on $[r, u) \quad P$-a.s.

8.5.B. The $\sigma$-algebra $\mathscr{B}_{\Delta} \times \mathscr{B}$ is generated by functions $h^{s}(x)$ such that $h^{s}\left(X_{s}\right)$ is right continuous on $\Delta$ for all $\omega$.

Obviously 1.7.A holds if $X$ is right and 1.7.B holds if $\xi$ is right.

8.6. Theorem 8.3. Suppose that $p$ is a Feller transition function and that the corresponding semigroup $T_{u}^{r}$ is continuous and satisfies the following condition:

8.6.A. For every $f \in C_{0}(E)$ and every $t \in \Delta, T_{u}^{r} f(x) \rightarrow 0$ as $r, u \rightarrow t$, $x \rightarrow \partial$.

Then there exists a right Markov process $X_{t}$ with transition function $p$.

Proof. Using Theorems 6.2 and 6.3 in Dynkin (1960), we show that $p$ is the transition function of a Markov process whose paths are right continuous with left limits. Let $\rho$ be a metric which determines the topology in $E$ and let $V_{\varepsilon}(x)=\{y: \rho(x, y) \geq \varepsilon\}$. We need to check that, for every $t \in \Delta$ and every compact set $\Gamma$,

$$
\sup _{x \in \Gamma} p\left(r, x ; u, V_{\varepsilon}(X)\right) \rightarrow 0 \quad \text { as } r<u \text { tend to } t
$$

and

$$
p(r, x ; u, \Gamma) \rightarrow 0 \text { as } x \rightarrow \partial, r<u \text { tend to } t .
$$

Put

$$
f_{x}(y)= \begin{cases}1-\rho(x, y) / \varepsilon & \text { if } \rho(x, y)<\varepsilon, \\ 0 & \text { otherwise }\end{cases}
$$

Note that $\mid f_{x}-f_{y} \| \leq \rho(x, y) / \varepsilon$. For every $\delta>0$, there exists a finite set $\Lambda=\left\{x_{1}, \ldots, x_{k}\right\} \subset \Gamma$ such that $\rho(x, \Lambda)<\delta$ for all $x \in \Gamma$. We have

$$
\begin{aligned}
p\left(r, x ; u, V_{\varepsilon}(x)\right) & \leq 1-T_{u}^{r} f_{x}(x)=f_{x}(x)-T_{u}^{r} f_{x}(x) \\
& \leq 2\left\|f_{x}-f_{x_{i}}\right\|+\left\|T_{u}^{r} f_{x_{i}}-f_{x_{i}}\right\| \\
& <2 \delta+\max _{i}\left\|T_{u}^{r} f_{x_{i}}-f_{x_{i}}\right\|
\end{aligned}
$$


which implies (8.4). To prove (8.5), we put $F=\{x: \rho(x, \Gamma)>\varepsilon\}$ and we note that $p(r, x ; u, \Gamma) \leq T_{u}^{r} g(x)$ where $g(x)=\rho(x, F) /[\rho(x, F)+\rho(x, \Gamma)]$.

Obviously, condition 8.5.B is satisfied. To check 8.5.A, we note that, by Theorem 5.9 in Dynkin (1960), for all $r<u \in \Delta, f \in B^{+}, P \in \mathscr{P}_{r}$ and for every stopping time $\tau$ relative to the filtration $\mathscr{F}{ }_{t}=\mathscr{F}[r, t]$,

$$
P\left\{f\left(X_{u}\right) \mid \mathscr{F}_{\tau}\right\}=P_{\tau, X_{\tau}} f\left(X_{u}\right) P \text {-a.s. on }\{\tau<u\}
$$

(the strong Markov property). This implies

$$
P\left[P_{\tau, X_{\tau}} f\left(X_{u}\right)\right]=P\left[1_{\tau<u} f\left(X_{u}\right)\right] .
$$

Since the process $Y_{t}=P_{t, X_{t}} f\left(X_{u}\right)$ is optional, its right continuity, $P$-a.s., follows from the relation $P Y_{\tau_{n}} \rightarrow P Y_{\tau}$ for every nonincreasing sequence of stopping times $\tau_{n}$ and for $\tau=\lim \tau_{n}$ (see Theorem 28 in Chapter 4, Dellacherie (1972)).

8.7. Theorem 8.4. Let a transition function $p$ satisfy the conditions of Theorem 8.3. Then the corresponding supertransition function $q$ also satisfies these conditions.

Proof. By Theorems 8.1 and 8.2, $q$ is Feller and the corresponding semigroup is continuous. To check that $q$ satisfies $8.6 . \mathrm{A}$, it is sufficient to prove that, for all $f \in W$,

$$
P_{r, \nu} F_{f}\left(X_{u}\right) \rightarrow 0, \quad \text { as } r, u \rightarrow t \in \Delta, \nu \rightarrow \partial .
$$

By (1.3), this is equivalent to the relation

$$
\left\langle V_{u}^{r} f, \nu\right\rangle \rightarrow+\infty \quad \text { as } r, u \rightarrow t \in \Delta, \nu \rightarrow \partial .
$$

By 2.2.B, there exists a $\beta>0$ such that $f(x) \geq \beta$ for all $x \in E$. It is clear from Theorem 3.1 and Lemma 3.1, that $V_{u}^{r} f \geq V_{u}^{r} \beta$. We note that

$$
V_{u}^{r} \beta=\beta[1+\beta(u-r)]^{-1}
$$

satisfies (1.2). Obviously, this implies (8.6).

\section{REFERENCES}

D. A. Dawson (1975), Stochastic evolution equations and related measure processes, J. Multivariate Anal. 3, 1-52.

(1977), The critical measure diffusion process, Z. Wahrsch. Verw. Gebiete 40, 125-145.

C. Dellacherie (1972), Capacités et processus stochastiques, Springer-Verlag, Berlin, Heidelberg and New York.

C. Dellacherie and P. A. Meyer (1983), Probabilités et potentiel: Théorie discrète du potentiel, Hermann, Paris.

E. B. Dynkin (1960), Theory of Markov processes, Pergamon Press, Oxford, London, New York and Paris.

(1972), Integral representation of excessive measures and excessive functions, Uspekhi Mat. Nauk 28, 36-64. Reprinted in London Mathematical Society Lecture Notes Series 54, pp. 145186, Cambridge Univ. Press, London and New York.

(1978), Sufficient statistics and extreme points, Ann. Probab. 6, 706-736. 
(1988), Representation for functionals of superprocesses by multiple stochastic integrals, with applications to self-intersection local times, Astérisque 157-158, 147-171.

E. B. Dynkin and S. E. Kuznetsov (1974), Determining functions of Markov processes and corresponding dual regular classes, Soviet Math. Dokl. 15, 20-23.

I. Iscoe (1986), A weighted occupation time for a class of measure-valued branching processes, Probab. Theory Related Fields 71, 85-116.

(1986a), Ergodic theory and local occupation time for measure-valued critical branching Brownian motion, Stochastics 18, 197-243.

P.-A. Meyer (1968), Probability and potential, Blaisdell, Waltham, Mass., Toronto and London.

S. Roelly-Coppoletta (1986), A criterion of convergence of measure-valued processes: application to measure branching processes, Stochastics 17, 43-65.

S. Watanabe (1968), A limit theorem of branching processes and continuous state branching processes, J. Math. Kyoto Univ. 8, 141-167.

J. B. Walsh (1986), An introduction to stochastic partial differential equations, École d'Été de Probabilités de Saint-Flour XIV-1984, Lecture Notes in Math., vol. 1180, Springer, Berlin, Heidelberg, New York and Tokyo, pp. 266-437.

Department of Mathematics, White Hall, Cornell University, Ithaca, New York 14853-7901 